\title{
NUMERICAL APPROXIMATIONS TO A NEW PHASE FIELD MODEL FOR TWO PHASE FLOWS OF COMPLEX FLUIDS
}

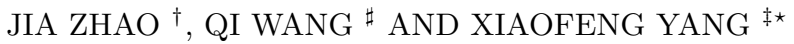

\begin{abstract}
We derive a new phase field theory for immiscible mixtures of nematic liquid crystals and viscous fluids using the variational principle coupled with the generalized Onsager principle. A novel phase transition mechanism is implemented to couple the nematic liquid crystal phase with the viscous fluid phase to arrive at the dissipative hydrodynamic model for incompressible fluid mixtures. Through a delicate explicit-implicit numerical discretization, we develop a decoupled, linear scheme for a simplified version of the phase field model, as well as a coupled, nonlinear scheme for the full model. Both schemes are shown as unconditionally energy stable with consistent, discrete dissipative energy laws. Several numerical examples are presented to show the effectiveness of the new model and the new numerical schemes developed for it.
\end{abstract}

\section{INTRODUCTION}

Liquid crystal droplets immersed in another fluid matrix or another liquid droplet immersed in liquid crystal matrices have many very interesting technological applications, like polymer stabilized liquid crystal dispersions for display devices, as a hosting and sensing platform for developing immunoassays, and as detecting devices for avidin-biotin binding etc. [1,8,24]. For the immiscible binary fluid mixture, the traditionally theoretical treatment is to develop models for each distinct fluid phase and the interfacial conditions between the phases. This is known as the sharp interface approach. Advantage of this approach is that one has a well-defined single phase model for each fluid phase involved in the mixture, and the interfacial condition can be derived based on momentum, mass, and energy conservation across the interface. When it comes to solve the models numerically, one has developed front tracking, level set and volumeof-fluid methods to deal with the computational challenge in these models. For 3-dimensional problems involving complex topological changes at the interface, these approaches could be very difficult to implement efficiently. Alternatively, one can consider the fluid mixture as one fluid with multiple components [2], in which the interface between distinct fluid phases is treated using a completely mixed transition fluid layer with a finite thickness. This modeling approach yields what is known as the diffuse interface model or phase field model.

Key words and phrases. Phase-field theory, Liquid crystals, Navier-Stokes equation, Cahn-Hilliard equation, Stability.

$\dagger$ Department of Mathematics, University of South Carolina, Columbia, SC 29208; Email: zhao62@math.sc.edu. \# Department of Mathematics, University of South Carolina, Columbia, SC 29208; Beijing Computational Science Research Center, Beijing 100091, China; School of Materials Science and Engineering, Nankai University, Tianjin 300071, China; Email: qwang@math.sc.edu.

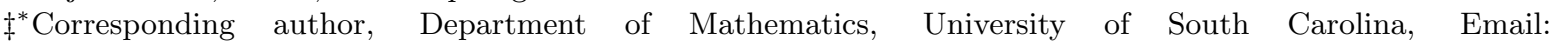
xfyang@math.sc.edu. 
The phase field model is an effective tool to incorporate the motion of free interfaces between multiple material components to hydrodynamics of the bulk components, whose origin can be traced back to Rayleigh [36] and Van der Waals [50]. With the new development in advanced algorithms and computational technologies recently, it has emerged as an efficient modeling and computational tool to resolve complex topology of the interface between different material components, for instances, vesicle membranes [6,9-11,30], crystal growth $[12,13]$, crack propagation [32,46], and multiphasic fluids flows [25, 29,31], etc.

The phase-field approach shows its effectiveness when the underlying model is derived from a variational principle following the generalized Onsager principle $[55,56]$. These models satisfy energy dissipation laws, making it possible for performing rigorous mathematical analyses as well as for designing property preserving numerical schemes that ensure the energy stability and accuracy in numerical simulations of the models [5, 20, 22, 29, 43, 45, 47, 55-57]. For more details about the development of numerical schemes for phase field models, readers are referred to some recent review articles on this topics $[25,44]$ and the references therein.

In this paper, we use phase field approaches to model and simulate the immiscible mixture of nematic liquid crystals (LCs) and viscous fluids. We recall that such phase-field models have been derived and used to make predictions previously [52-54,57-59,62-64], where the elastic energy of liquid crystal is coupled with the phase field variable (as a labeling function) through a nonlinear "weight" function in the free energy. The coupling allows the elastic energy associated with the liquid crystal only contributes in the LC phase, but not in the viscous fluid phase. While the resulted governing equations and their corresponding numerical schemes follow the continuous and discrete dissipative energy laws, respectively, such couplings can yield singular terms in the governing system of equations, thereby creating potential difficulties in numerical simulations. Specifically, (i). the singularity may appear due to the fact that the nonlinear weight function may vanish in the viscous fluid phase; (ii). the liquid crystal director field may not vanish completely in the viscous fluid phase due to the numerical truncation, making the viscous fluid phase a weakly liquid crystal fluid; (iii). the model ends up with the Laplace operator of variable coefficients that are close to zero in some region of the domain, which in turn leads to the consequence that one has to use fine spatial mesh and time step as well as delicate iterative methods to control the propagation of the error.

Inspired by the work in [49], we develop a novel strategy based on the "phase transition" mechanism to reformulate this mixture fluid model. Namely, we introduce a phase transition mechanism in the bulk energy of the liquid crystal phase to relax the otherwise rigid constraint on the director field in the liquid crystal theory, such as the classical Ericksen-Leslie theory, such that the liquid crystal elastic energy reduces to the Oseen-Frank elastic energy in the defect free liquid crystal region, admits possible defects, and essentially vanishes in the viscous fluid phase. Such a phase transition mechanism on the low order bulk energy not only work as a penalizing term to release the unit vector constraint on the director field, but also avoid introducing any singularities in the Laplace operator. In addition, to solve the model derived from variational principles, we can combine several approaches that have been proven efficient for the phase transport equation and for the Navier-Stokes equation altogether, which include the stabilizing method (cf. $[6,41,42,52,60]$ ) for phase transport equations, and the projectionmethod (cf. $[18,19,41,60])$ for the Navier-Stokes equation.

In this paper, we develop a linear, decoupled scheme for a simplified model of liquid crystalviscous fluid mixture where the velocity gradient is weak. We show that this scheme satisfies a 
discrete energy dissipation law and is unconditionally energy stable. For the full hydrodynamic phase field model, we devise a coupled, nonlinear time discretization scheme and prove that it is unconditionally energy stable as well. We then apply the coupled scheme, modified slightly using extrapolation to decouple some of the nonlinear terms, to benchmark the full hydrodynamic model as well as the numerical scheme on three numerical examples. The three examples include a study on the viscous drop shape while it is embedded in a liquid crystal matrix, a dripping liquid crystal drop from a faucet, and a liquid crystal filament breakup due to capillary instability. The numerical studies demonstrate that this new model can capture the phenomena predicted by the previous models in $[41,53,60]$, give better results at the interface and in the viscous fluid region, and predict results consistent with the experimental findings in [48] qualitatively.

The rest of the paper is organized as follows. In Section 2, we derive the new phasefield model for the mixture of nematic liquid crystals and viscous fluids using the variational principle coupled with the generalized Onsager principle $[55,56]$, and show the model obeys an energy dissipation law. In Section 3, we develop a linear, decoupled, energy stable numerical scheme for a simplified model and a coupled energy stable scheme for the full model. In Section 4 , we present the mesh refinement test and three numerical examples to illustrate the accuracy and efficiency of the proposed models and schemes. The concluding remarks are given in Section 5 .

\section{Phase Field model For mixtures of Nematic Liquid CRYSTAls AND Viscous fluids}

We consider a phase field model for immiscible mixtures of nematic liquid crystals (LC) immersed in a viscous fluid matrix. Consider a smooth domain $\Omega \subset \mathbb{R}^{d}, d=2,3$. We use a phase field variable $\phi: \Omega \rightarrow \mathbb{R}$ to denote the volume fraction of the LC fluid (fluid I) in the mixture, $\forall \boldsymbol{x} \in \Omega$,

$$
\phi(\boldsymbol{x}, t)= \begin{cases}1, & \mathbf{x} \text { in fluid I (LC fluid), } \\ 0, & \mathbf{x} \text { in fluid II (viscous fluid). }\end{cases}
$$

There exists a layer $\{\mathbf{x} \mid 0<\phi<1\}$, connecting the LC phase $(\phi=1)$ with the viscous fluid phase $(\phi=0)$. We define technically the interface between the two phases at surface $\{\boldsymbol{x}: \phi(\boldsymbol{x}, t)=0.5\}$.

The interfacial free energy, defined in the entire domain, is defined as follows:

$$
E_{m}=\int_{\Omega} \lambda\left(\frac{\varepsilon}{2}|\nabla \phi|^{2}+F(\phi)\right) d \boldsymbol{x}
$$

where $F(\phi)=\frac{1}{\varepsilon} \phi^{2}(1-\phi)^{2}$ is a Ginzburg-Landau double well potential, $\lambda$ measures the strength of the interfacial energy density, which is proportional to the surface tension [57]. The first term represents the conformational entropy, promoting material mixing, while the double well bulk energy density represents the hydrophobic interaction, promoting phase separation. It is the competition between the opposing effects that allows the coexistence of two distinct phases mediated through a transitional layer whose thickness is controlled by the model parameter $\varepsilon$.

For the nematic LC phase, we adopt the Oseen-Frank distortional elastic energy density with the one constant approximation $K_{1}=K_{2}=K_{3}=K[14,15,26]$, i.e.,

$$
E_{b}(\boldsymbol{d})=\frac{K}{2}|\nabla \boldsymbol{d}|^{2}
$$


where $K$ is the Frank elastic constant and unit vector $\boldsymbol{d}(|\boldsymbol{d}|=1)$ is the director field representing the orientation of the LC molecules. Note that, for the binary mixture, the elastic energy of LCs only exists in the LC phase. In order to associate the elastic energy only to the LC phase, we introduce a phase transition mechanism to switch the potential on only in the LC phase following the idea introduced in [49]. We then augment the Oseen-Frank elastic energy by a bulk term as follows:

$$
E_{b}^{\text {trans }}(\boldsymbol{d}, \phi)=K \int_{\Omega}\left(\frac{1}{2}|\nabla \boldsymbol{d}|^{2}+G(\boldsymbol{d}, \phi)\right) d \boldsymbol{x}, \text { with } G(\boldsymbol{d}, \phi)=\frac{|\boldsymbol{d}|^{2}\left(|\boldsymbol{d}|^{2}-\mathbf{\Psi}(\phi)\right)}{4 \eta^{2}}
$$

where $\boldsymbol{\Psi}(\phi)$ is a monotonically decreasing function with $\boldsymbol{\Psi}(1)=1>0$ and $\boldsymbol{\Psi}(0)<0$, which controls the phase transition between the LC phase and the viscous phase, and $\eta$ is a model parameter controlling the size of the defect core $(|\boldsymbol{d}|=0)$. In the pure LC phase where $\phi=1$, bulk energy $\frac{K}{4 \eta^{2}}|\boldsymbol{d}|^{2}\left(|\boldsymbol{d}|^{2}-1\right)$ warrants $|\boldsymbol{d}|=1$ so that the effective elastic energy is given by the Oseen-Frank distortional energy. Whereas, in the viscous phase where $\phi=0$, the minimization of the bulk energy prefers $|\boldsymbol{d}|=0$, which effectively annihilates the entire elastic energy contribution.

By adding this penalty term $G(\boldsymbol{d}, \phi)$, we literally relax the constraint on $(|\boldsymbol{d}|=1)$ to allow it to be zero and turn $|\boldsymbol{d}|$ into an order parameter for the degree of orientation of $\boldsymbol{d}$. When $|\boldsymbol{d}|=0$, we claim the LC phase is isotropic and nematic otherwise. The phase transition mechanism enables us to effectively control the director $\boldsymbol{d}$ to be zero in the viscous phase so as to the LC elastic energy can only make a nontrivial contribution to the LC phase.

Comparing to an alternative way, i.e., the nonlinear coupling approach to limiting the elastic energy to the LC phase alone by weighting the Oseen-Frank elastic energy using the phase variable $\phi$ or $\phi^{2}$ in some previous papers $[17,21,27,28,53,57,58,60]$, this phase transition approach avoids the singular Laplacian to arrive at a more regular governing system of equations for the mixture system. It thereby eliminates the potential instability associated with the singular Laplacian. In the transition layer where $0<\phi<1$, the addition of this new bulk energy term introduces a demixing mechanism to promote phase separation between the LC phase and the viscous fluid phase.

At the interface between the LC and the viscous fluid phase, LC molecules may prefer some orientation known as the easy (anchoring) direction. There are two types of commonly used anchoring conditions: the planar (or parallel) anchoring, where all directions in the tangent plane of the interface are easy directions, and the homeotropic (or normal) anchoring, where the easy direction is the normal to the interface $[17,53,57,58,60]$. The anchoring condition can be modeled using a surface free energy functional known as the anchoring energy. We propose an anchoring energy that can accommodate both the parallel and homeotropic anchoring as follows [58].

$$
E_{a}=\int_{\Omega}\left(\frac{A_{1}}{2}(\boldsymbol{d} \cdot \nabla \phi)^{2}+\frac{A_{2}}{2}\left(|\boldsymbol{d}|^{2}|\nabla \phi|^{2}-(\boldsymbol{d} \cdot \nabla \phi)^{2}\right)\right) d \boldsymbol{x},
$$

where $A_{1}, A_{2}$ are positive model parameters that control the strength of planar and homeotropic anchoring, respectively.

The total energy of the binary mixture system is given by the sum of the kinetic energy $E_{k}$, the mixing energy $E_{m}$, the LC elastic energy $E_{b}^{\text {trans }}$, and the anchoring energy $E_{a}$ :

$$
E=E_{k}+E_{m}+E_{b}^{\text {trans }}+E_{a},
$$


where $E_{k}=\int_{\Omega} \frac{1}{2} \rho|\boldsymbol{u}|^{2} d \boldsymbol{x}, \rho$ is volume-averaged the density of the mixture system, $\boldsymbol{u}$ is the volume-averaged velocity field.

In this paper, we consider the case where the two fluid components have matching density and viscosity, i.e., $\rho_{1}=\rho_{2}=1$ and $\nu_{1}=\nu_{2}=\nu$ for simplicity. Following the generalized Onsager's principle $[34,35,55,56]$, we derive the following governing system of equations and present them in dimensionless forms:

$$
\begin{aligned}
& \boldsymbol{d}_{t}+\boldsymbol{u} \cdot \nabla \boldsymbol{d}-\boldsymbol{W} \cdot \boldsymbol{d}-a \boldsymbol{D} \cdot \boldsymbol{d}=-M_{1} \boldsymbol{\mu}_{\mathbf{1}}, \quad \boldsymbol{\mu}_{\mathbf{1}}=\frac{\delta E}{\delta \boldsymbol{d}}, \\
& \phi_{t}+\nabla \cdot(\boldsymbol{u} \phi)=M_{2} \Delta \mu_{2}, \quad \mu_{2}=\frac{\delta E}{\delta \phi}, \\
& \boldsymbol{u}_{t}+\boldsymbol{u} \cdot \nabla \boldsymbol{u}=-\nabla p+\nabla \cdot(2 \nu \boldsymbol{D})-\phi \nabla \mu_{2}+\boldsymbol{\mu}_{1} \nabla \boldsymbol{d}+\nabla \cdot\left(\frac{a-1}{2} \boldsymbol{d} \boldsymbol{\mu}_{1}+\frac{a+1}{2} \boldsymbol{\mu}_{1} \boldsymbol{d}\right), \\
& \nabla \cdot \boldsymbol{u}=0,
\end{aligned}
$$

where $p$ is the hydrostatic pressure, $M_{1}$ is inversely proportional to the relaxation time of the LCs, $M_{2}$ the mobility for transporting the phase variable $\phi, \boldsymbol{D}=\frac{1}{2}\left(\nabla \boldsymbol{u}+(\nabla \boldsymbol{u})^{T}\right)$ is the rate of strain tensor, $\boldsymbol{W}=\frac{1}{2}\left(\nabla \boldsymbol{u}-(\nabla \boldsymbol{u})^{T}\right)$ is the vorticity tensor, $a$ is a geometry parameter of the liquid crystal molecule and $\nu$ is the solvent viscosity. Here,

$$
\begin{aligned}
\boldsymbol{\mu}_{1} & =K\left(-\Delta \boldsymbol{d}+\frac{1}{\eta^{2}} \boldsymbol{d}\left(|\boldsymbol{d}|^{2}-\frac{\boldsymbol{\Psi}(\phi)}{2}\right)\right) \\
& -A_{1}(\boldsymbol{d} \cdot \nabla \phi) \nabla \phi-A_{2}\left(|\nabla \phi|^{2} \boldsymbol{d}-(\boldsymbol{d} \cdot \nabla \phi) \nabla \phi\right),
\end{aligned}
$$

and

$$
\begin{aligned}
\mu_{2}= & \lambda(-\varepsilon \Delta \phi+f(\phi))-\frac{1}{4 \eta^{2}} \boldsymbol{\Psi}^{\prime}(\phi)|\boldsymbol{d}|^{2} \\
& -A_{1} \nabla \cdot((\boldsymbol{d} \cdot \nabla \phi) \boldsymbol{d})-A_{2}\left(\nabla \cdot\left(|\boldsymbol{d}|^{2} \nabla \phi\right)-\nabla \cdot((\boldsymbol{d} \cdot \nabla \phi) \boldsymbol{d})\right),
\end{aligned}
$$

where $f(\phi)=F^{\prime}(\phi)=\frac{2}{\varepsilon} \phi(1-\phi)(1-2 \phi) . \quad-\boldsymbol{\mu}_{\mathbf{1}}$ is the molecular field in the liquid crystal theory and $\mu_{2}$ is the generalized chemical potential.

Remark 2.1. We use index notations for several cases to better explain the operators. In (2.7)-(2.10), $(\nabla \boldsymbol{u})_{i j}=\partial_{j} \boldsymbol{u}_{i},\left(\boldsymbol{d} \boldsymbol{\mu}_{1}\right)_{i j}=\boldsymbol{d}_{i}\left(\boldsymbol{\mu}_{1}\right)_{j},\left(\boldsymbol{\mu}_{1} \boldsymbol{d}\right)_{i j}=\left(\boldsymbol{\mu}_{1}\right)_{i} \boldsymbol{d}_{j}$ and $\left(\boldsymbol{\mu}_{1} \nabla \boldsymbol{d}\right)_{i}=\left(\boldsymbol{\mu}_{1}\right)_{j} \partial_{i} \boldsymbol{d}_{j}$.

Remark 2.2. The term $-\boldsymbol{W} \cdot \boldsymbol{d}-a \boldsymbol{D} \cdot \boldsymbol{d}$ in $(2.7)$ is due to the frame invariant time derivative of vector $\boldsymbol{d}$, which induces an extra elastic stress $\frac{a-1}{2} \boldsymbol{d} \boldsymbol{\mu}_{1}+\frac{a+1}{2} \boldsymbol{\mu}_{1} \boldsymbol{d}$ in (2.9) [56] in the system.

Alternatively, one can choose the Allen-Cahn dynamics for the phase transport equation, namely,

$$
\phi_{t}+(\boldsymbol{u} \cdot \nabla) \phi=-M_{2} \mu_{2} .
$$

In the Allen-Cahn equation, $M_{2}$ is inversely proportional to the relaxation time of phase dynamics. In spite of the fact that the volume is not conserved in the Allen-Cahn equation, one can preserve the volume by using a Lagrange multiplier or modify the free energy by adding a volume penalty term (cf. $[29,38]$ ). In this paper, we adopt the Cahn-Hilliard model and remark that all the analytical and numerical work to follow can be generalized to the Allen-Cahn model in a straightforward fashion. 
Throughout the paper, we use the following boundary conditions:

$$
\left.\boldsymbol{u}\right|_{\partial \Omega}=0,\left.\quad \nabla \phi \cdot \boldsymbol{n}\right|_{\partial \Omega}=0,\left.\quad \boldsymbol{n} \cdot \nabla \mu\right|_{\partial \Omega}=0,\left.\quad \boldsymbol{n} \cdot \nabla \boldsymbol{d}\right|_{\partial \Omega}=0
$$

with $\boldsymbol{n}$ the unit outward normal of the domain boundary $\partial \Omega$. These conditions warrant the boundary effect does not contribute to the energy dissipation. In fact, since periodic boundary conditions also have no contributions to the bulk energy dissipation, all results presented in this paper are valid for periodic boundary conditions as well.

Since the equations are derived via the generalized Onsager principle, the total energy of the system (2.7)-(2.10) is dissipative. The energy dissipation rate is obtained as follows

$$
\begin{aligned}
\frac{d E}{d t}= & \int_{\Omega}\left(\frac{\delta E}{\delta \boldsymbol{u}} \cdot \boldsymbol{u}_{t}+\frac{\delta E}{\delta \phi} \phi_{t}+\frac{\delta E}{\delta \boldsymbol{d}} \cdot \boldsymbol{d}_{t}\right) d \boldsymbol{x} \\
= & \int_{\Omega} \boldsymbol{u} \cdot\left(-\boldsymbol{u} \cdot \nabla \boldsymbol{u}-\nabla p+\nu \nabla \cdot \boldsymbol{D}-\phi \nabla \mu_{2}+\boldsymbol{\mu}_{1} \nabla \boldsymbol{d}+\nabla \cdot\left(\frac{a}{2}\left(\boldsymbol{d} \boldsymbol{\mu}_{1}+\boldsymbol{\mu}_{1} \boldsymbol{d}\right)-\frac{1}{2}\left(\boldsymbol{d} \boldsymbol{\mu}_{1}-\boldsymbol{\mu}_{1} \boldsymbol{d}\right)\right)\right) d \boldsymbol{x} \\
& +\int_{\Omega} \mu_{2}\left(-\nabla \cdot(\boldsymbol{u} \phi)+M_{2} \Delta \mu_{2}\right) d \boldsymbol{x}+\int_{\Omega} \boldsymbol{\mu}_{1}\left(-\boldsymbol{u} \cdot \nabla \boldsymbol{d}+\boldsymbol{W} \cdot \boldsymbol{d}+a \boldsymbol{D} \cdot \boldsymbol{d}-M_{1} \boldsymbol{\mu}_{1}\right) d \boldsymbol{x} \\
= & \int_{\Omega}-\left(\nu|\nabla \boldsymbol{u}|^{2}+M_{1}\left|\boldsymbol{\mu}_{\mathbf{1}}\right|^{2}+M_{2}\left|\nabla \mu_{2}\right|^{2}\right) d \boldsymbol{x} \\
& +\int_{\Omega}\left(-\frac{a}{2}\left(\boldsymbol{d} \boldsymbol{\mu}_{1}+\boldsymbol{\mu}_{1} \boldsymbol{d}\right) \nabla \boldsymbol{u}+\frac{1}{2}\left(\boldsymbol{d} \boldsymbol{\mu}_{1}-\boldsymbol{\mu}_{1} \boldsymbol{d}\right) \nabla \boldsymbol{u}\right) d \boldsymbol{x}+\int_{\Omega} \boldsymbol{\mu}_{1}(\boldsymbol{W} \cdot \boldsymbol{d}+a \boldsymbol{D} \cdot \boldsymbol{d}) d \boldsymbol{x} .
\end{aligned}
$$

It is easy to check

$$
\begin{gathered}
\int_{\Omega} \boldsymbol{\mu}_{1}(\boldsymbol{W} \cdot \boldsymbol{d}) d \boldsymbol{x}+\int_{\Omega} \frac{1}{2}\left(\boldsymbol{d} \boldsymbol{\mu}_{\mathbf{1}}-\boldsymbol{\mu}_{\mathbf{1}} \boldsymbol{d}\right) \nabla \boldsymbol{u} d \boldsymbol{x}=0 \\
\int_{\Omega} \boldsymbol{\mu}_{1}(\boldsymbol{D} \cdot \boldsymbol{d}) d \boldsymbol{x}-\int_{\Omega} \frac{1}{2}\left(\boldsymbol{d} \boldsymbol{\mu}_{\mathbf{1}}+\boldsymbol{\mu}_{\mathbf{1}} \boldsymbol{d}\right) \nabla \boldsymbol{u} d \boldsymbol{x}=0 .
\end{gathered}
$$

Thus we obtain the energy dissipation law as follows

$$
\frac{d E}{d t}=-\int_{\Omega}\left(\nu|\nabla \boldsymbol{u}|^{2}+M_{1}\left|\boldsymbol{\mu}_{\mathbf{1}}\right|^{2}+M_{2}\left|\nabla \mu_{2}\right|^{2}\right) d \boldsymbol{x}
$$

We note that the energy dissipation law in fact acts as a guideline for us to develop stable and efficient numerical schemes in the following. However, the process obtaining the PDE energy law involves the inner product with $\boldsymbol{\mu}_{\mathbf{1}}$ or $\mu_{2}$ that actually includes the second order derivatives of $\boldsymbol{d}$ or $\phi$, respectively. And it is not convenient to use them as test functions in numerical approximations, making it difficult to prove the discrete energy dissipation law. To circumvent the problem, we reformulate the system (2.7)-(2.10) in an alternative form which 
is convenient for numerical approximations.

$$
\begin{aligned}
& \frac{\dot{\boldsymbol{d}}}{M_{1}}=K\left(\Delta \boldsymbol{d}-\frac{1}{\eta^{2}} \boldsymbol{d}\left(|\boldsymbol{d}|^{2}-\frac{\boldsymbol{\Psi}(\phi)}{2}\right)\right) \\
& +A_{1}(\boldsymbol{d} \cdot \nabla \phi) \nabla \phi+A_{2}\left(|\nabla \phi|^{2} \boldsymbol{d}-(\boldsymbol{d} \cdot \nabla \phi) \nabla \phi\right), \\
& \phi_{t}+\nabla \cdot(\boldsymbol{u} \phi)=M_{2} \Delta \mu_{2}, \\
& \mu_{2}=\lambda(-\varepsilon \Delta \phi+f(\phi))-\frac{K}{4 \eta^{2}} \boldsymbol{\Psi}^{\prime}(\phi)|\boldsymbol{d}|^{2} \\
& -A_{1} \nabla \cdot((\boldsymbol{d} \cdot \nabla \phi) \boldsymbol{d})-A_{2}\left(\nabla \cdot\left(|\boldsymbol{d}|^{2} \nabla \phi\right)-\nabla \cdot((\boldsymbol{d} \cdot \nabla \phi) \boldsymbol{d})\right), \\
& \boldsymbol{u}_{t}+\boldsymbol{u} \cdot \nabla \boldsymbol{u}=-\nabla p+\nabla \cdot(\nu \boldsymbol{D}) \\
& -\phi \nabla \mu_{2}-\frac{\dot{\boldsymbol{d}}}{M_{1}} \nabla \boldsymbol{d}-\nabla \cdot\left(\frac{1-a}{2} \boldsymbol{d} \dot{\boldsymbol{d}}-\frac{1+a}{2} \dot{\boldsymbol{d}} \boldsymbol{d}\right), \\
& \nabla \cdot \boldsymbol{u}=0
\end{aligned}
$$

where

$$
\dot{\boldsymbol{d}}=\boldsymbol{d}_{t}+\boldsymbol{u} \cdot \nabla \boldsymbol{d}-\boldsymbol{W} \cdot \boldsymbol{d}-a \boldsymbol{D} \cdot \boldsymbol{d} .
$$

With this new formulation, the energy dissipation law is rewritten as follows:

$$
\frac{d E}{d t}=-\int_{\Omega}\left(\nu|\nabla \boldsymbol{u}|^{2}+\frac{1}{M_{1}}|\dot{\boldsymbol{d}}|^{2}+M_{2}\left|\nabla \mu_{2}\right|^{2}\right) d \boldsymbol{x} .
$$

Remark 2.3. The difference between (2.7)-(2.10) and (2.18)-(2.22) is that we replace $\boldsymbol{\mu}_{\mathbf{1}}$ by $-\frac{\dot{d}}{M_{1}}$ in the momentum equation (2.21). We emphasize that the above derivation is suitable in a finite dimensional approximation since test function $\boldsymbol{d}_{t}$ is in the same subspaces as $\boldsymbol{d}$. Hence, it allows us to design numerical schemes that satisfy discrete energy laws.

\section{Numerical Approximations: Energy Stable Schemes}

One of the desirable properties that one would like to preserve for the discretized dissipative system is to maintain its own energy dissipation law that is consistent with the energy law obeyed by the continuous partial differential equation system. Practically, this is an indication for a good approximation to the continuous system. This type of numerical schemes is known as the energy stable scheme. In the following, we will design a semi-discrete energy stable scheme that addresses the following issues:

- the coupling of the velocity and the pressure through the incompressible condition;

- the stiffness in the phase field equation and the director equation associated with the interfacial width $\varepsilon$ and the parameter $\eta$, where $\varepsilon, \eta$ are much smaller than the size of the computational domain;

- the nonlinear couplings among the momentum transport equation, the phase transport equation and the director equation.

There are two popular approaches to handle the non-convex GinzburgLandau potential $F(\phi)$ in (2.2). One is the convex splitting method (cf. $[16,37]$ ), another is the stabilization method (cf. [41, 42,61]). Here we adopt the latter one, which avoid solving a nonlinear equation.

In doing so, we have to put some constraints on the choice of the potential function $F(\phi)$, i.e., it must satisfy the following conditions:

- (i) $F(x)$ has continuous second order derivatives; 
- (ii) there exists a positive constant $L_{F}$ such that

$$
\max _{x \in \mathbb{R}}\left|F^{\prime \prime}(x)\right| \leq L_{F} .
$$

One immediately notices that condition (ii) is not satisfied by both double-well potentials $F(\phi)$ and $G(\boldsymbol{d}, \phi)$. However, since it is well-known that the Allen-Cahn equation satisfies the maximum principle (for Cahn-Hilliard equation, a similar result was established in [4]), we can always truncate $F(\phi)$ to a quadratic growth outside an interval $[-M, M]$ without affecting the solution if the maximum norm of the initial condition $\phi_{0}$ is bounded by $M$. For instance, $M=1$ and

$$
\tilde{F}(\phi)=\left\{\begin{array}{l}
\frac{1}{\varepsilon} \phi^{2}, \quad \phi<0, \\
\frac{1}{\varepsilon} \phi^{2}(1-\phi)^{2}, \quad 0 \leq \phi \leq 1, \\
\frac{1}{\varepsilon}(1-\phi)^{2}, \quad \phi>1 .
\end{array}\right.
$$

Hence, it has been a common practice (cf. $[7,23,39])$ to consider the Allen-Cahn and the CahnHilliard equation with a truncated double-well potential $\tilde{F}(\phi)$ instead of the originally defined one.

For $G(\boldsymbol{d}, \phi)$, we note that we can choose $\boldsymbol{\Psi}(\phi)$ freely as long as it is a monotonically increasing function for $\phi$ satisfying $\boldsymbol{\Psi}(1)=1>0$ and $\boldsymbol{\Psi}(0)<0$. For instance, we can use

$$
\boldsymbol{\Psi}(\phi)=\tanh \frac{\phi-0.5}{\varepsilon} \text {, or } \boldsymbol{\Psi}(\phi)=\frac{1}{2}(\phi+1)^{2}-1 \text {, or } \boldsymbol{\Psi}(\phi)=2 \phi-1 \text {. }
$$

It is obvious that a complicated $\boldsymbol{\Psi}(\phi)$ may lead to a sophisticated $\boldsymbol{\Psi}^{\prime}(\phi)$ in the governing PDE system as well as the numerical scheme. In this paper, we choose $\boldsymbol{\Psi}(\phi)=2 \phi-1$ for simplicity. As the result, the nonlinear potential $G(\boldsymbol{d}, \phi)$ can be split into the addition of a fourth order polynomial and a second order polynomial as follows:

$$
\begin{aligned}
G(\boldsymbol{d}, \phi) & =G_{1}(\boldsymbol{d})+G_{2}(\boldsymbol{d}, \phi) \\
& =\frac{|\boldsymbol{d}|^{2}\left(|\boldsymbol{d}|^{2}-1\right)}{4 \eta^{2}}+\frac{|\boldsymbol{d}|^{2}(1-\phi)}{2 \eta^{2}} .
\end{aligned}
$$

Notice that the form of $G_{1}(\boldsymbol{d})$ is similar to the $F(\phi)$. Thus, we can modify this function outside a ball in $\{|\boldsymbol{d}|<1\} \in \mathbb{R}^{3}$ of radius 1 as follows:

$$
\eta^{2} \tilde{G}_{1}(\boldsymbol{d})= \begin{cases}|\boldsymbol{d}|^{4}-|\boldsymbol{d}|^{2}, & |\boldsymbol{d}| \leq 1 \\ (|\boldsymbol{d}|-1)^{2}, & |\boldsymbol{d}|>1\end{cases}
$$

Then, there exists a positive constant $L_{G}$ such that

$$
\max _{\boldsymbol{x} \in \mathbb{R}^{3}}\left|H_{\tilde{G}_{1}}(\boldsymbol{x})\right| \leq L_{G},
$$

where $\left(H_{\tilde{G}_{1}}(\boldsymbol{x})\right)_{i, j}=\frac{\partial^{2} \tilde{G}_{1}}{\partial x_{i} \partial x_{j}}, i, j=1,2,3$ is the Hessian matrix of $\tilde{G}_{1}(\boldsymbol{x})$. For simplicity, we drop $^{\sim}$ on $\tilde{F}$ and $\tilde{G}_{1}$ in the following.

Remark 3.1. Concerning the modification of the bulk potentials $F$ and $G$, one could simply stipulate the bulk potentials are given by the modified ones in the first place. These potentials are phenomenological in the model to enforce certain constraints on the range of the phase 
variable and the length of the director vector. Overly emphasizing their functional forms away from physically relevant ranges is meaningless physically since the state along that part of the modified potential or the original potential is always in a far from equilibrium transient state whose validity is beyond the capability of the model.

For scalar functions $u, v$ and vector functions $\boldsymbol{u}=\left(u_{1}, u_{2}, u_{3}\right)$ and $\boldsymbol{v}=\left(v_{1}, v_{2}, v_{3}\right)$, we define the $L^{2}$ inner product as follows:

$$
\begin{aligned}
& (u, v)=\int_{\Omega} u v d x, \quad\|u\|^{2}=(u, u), \\
& (\boldsymbol{u}, \boldsymbol{v})=\int_{\Omega} \boldsymbol{u} \boldsymbol{v}^{T} d \boldsymbol{x}=\sum_{i=1}^{3} \int_{\Omega} u_{i} v_{i} d \boldsymbol{x}, \quad\|\boldsymbol{u}\|^{2}=(\boldsymbol{u}, \boldsymbol{u}) .
\end{aligned}
$$

Now, we are ready to present our energy stable schemes.

3.1. Linear, Decoupled, Semi-Discrete Scheme for a Simplified Model. We consider a case where the anchoring effect as well as the velocity gradient are weak. Then, we set $A_{1}=A_{2}=0$ and neglect $\boldsymbol{W}$ and $\boldsymbol{D}$ in the director transport equation to obtain a simplified model as follows:

$$
\begin{aligned}
& \frac{\dot{\boldsymbol{d}}}{M_{1}}-K\left(\Delta \boldsymbol{d}-g_{1}(\boldsymbol{d})\right)=-\frac{K}{\eta^{2}} \boldsymbol{d}(1-\phi), \\
& \phi_{t}+\nabla \cdot(\boldsymbol{u} \phi)=M_{2} \Delta \mu_{2}, \\
& \mu_{2}=\lambda(-\varepsilon \Delta \phi+f(\phi))-\frac{K}{2 \eta^{2}}|\boldsymbol{d}|^{2}, \\
& \boldsymbol{u}_{t}+\boldsymbol{u} \cdot \nabla \boldsymbol{u}-\nu \Delta \boldsymbol{u}+\nabla p+\phi \nabla \mu_{2}+\frac{\dot{\boldsymbol{d}}}{M_{1}} \nabla \boldsymbol{d}=0 \\
& \nabla \cdot \boldsymbol{u}=0
\end{aligned}
$$

where $g_{1}(\boldsymbol{d})=G_{1}^{\prime}(\boldsymbol{d})$. For this model, we design an effective numerical scheme to solve it. The linear, decoupled, first order numerical scheme reads as follows.

Given the initial conditions $\boldsymbol{d}^{0}, \phi^{0}, \boldsymbol{u}^{0}$ and $p^{0}=0$ and having computed $\left(\boldsymbol{d}^{n}, \phi^{n}, \boldsymbol{u}^{n}, p^{n}\right)$ for $n \geq 0$, we compute $\left(\boldsymbol{d}^{n+1}, \phi^{n+1}, \tilde{\boldsymbol{u}}^{n+1}, \boldsymbol{u}^{n+1}, p^{n+1}\right)$ in the following sequence.

Step 1: Solve for $\boldsymbol{d}^{n+1}$,

$$
C^{n}\left(\boldsymbol{d}^{n+1}-\boldsymbol{d}^{n}\right)+\frac{1}{M_{1}} \dot{\boldsymbol{d}}^{n+1}-K\left(\Delta \boldsymbol{d}^{n+1}-g_{1}\left(\boldsymbol{d}^{n}\right)\right)=-\frac{K}{\eta^{2}} \boldsymbol{d}^{n+1}\left(1-\phi^{n}\right)
$$

subject to

$$
\begin{aligned}
& \left.\frac{\partial \boldsymbol{d}^{n+1}}{\partial \boldsymbol{n}}\right|_{\partial \Omega}=0, \\
& \dot{\boldsymbol{d}}^{n+1}=\frac{1}{\delta t}\left(\boldsymbol{d}^{n+1}-\boldsymbol{d}^{n}\right)+\boldsymbol{u}_{\star}^{n} \cdot \nabla \boldsymbol{d}^{n}, \\
& \boldsymbol{u}_{\star}^{n}=\boldsymbol{u}^{n}-\frac{1}{M_{1}} \delta t \dot{\boldsymbol{d}}^{n+1} \nabla \boldsymbol{d}^{n} .
\end{aligned}
$$


Step 2: Solve for $\phi^{n+1}$,

$$
\begin{aligned}
& \frac{1}{\delta t}\left(\phi^{n+1}-\phi^{n}\right)+\nabla \cdot\left(\boldsymbol{u}_{\star \star}^{n} \phi^{n}\right)=M_{2} \Delta \mu_{2}^{n+1}, \\
& \mu_{2}^{n+1}=S^{n}\left(\phi^{n+1}-\phi^{n}\right)+\lambda\left(-\varepsilon \Delta \phi^{n+1}+f\left(\phi^{n}\right)\right)-\frac{K}{2 \eta^{2}}\left|\boldsymbol{d}^{n+1}\right|^{2},
\end{aligned}
$$

subject to

Step 3: Solve for $\tilde{\boldsymbol{u}}^{n+1}$,

2) $\frac{1}{\delta t}\left(\tilde{\boldsymbol{u}}^{n+1}-\boldsymbol{u}^{n}\right)+\left(\boldsymbol{u}^{n} \cdot \nabla\right) \tilde{\boldsymbol{u}}^{n+1}-\nu \Delta \tilde{\boldsymbol{u}}^{n+1}+\nabla p^{n}+\phi^{n} \nabla \mu_{2}^{n+1}+\frac{1}{M_{1}} \dot{\boldsymbol{d}}^{n+1} \nabla \boldsymbol{d}^{n}=0$, subject to

$$
\left.\tilde{\boldsymbol{u}}^{n+1}\right|_{\partial \Omega}=0 .
$$

Step 4: Update $\boldsymbol{u}^{n+1}$ and $p^{n+1}$,

$$
\begin{aligned}
& \frac{\boldsymbol{u}^{n+1}-\tilde{\boldsymbol{u}}^{n+1}}{\delta t}=-\nabla\left(p^{n+1}-p^{n}\right), \\
& \nabla \cdot \boldsymbol{u}^{n+1}=0,\left.\quad \boldsymbol{u}^{n+1} \cdot \boldsymbol{n}\right|_{\partial \Omega}=0 .
\end{aligned}
$$

To better understand this new scheme, we list several features as follows.

- A pressure-correction scheme [18] is used to decouple the computation of the pressure from that of the velocity.

- For nonlinear terms $f(\phi)$ and $g_{1}(\boldsymbol{d})$, we recall that both have small parameters $(\varepsilon, \eta \ll$ 1) in the denominator, thus the explicit treatment of the terms usually leads to a severe restriction on time step $\delta t$. to circumvent the situation, we introduce ' stabilizing' terms, whose coefficients $C^{n}$ and $S^{n}$ are to be determined, to improve the stability. It allows us to treat the nonlinear term explicitly without suffering from any time step constraint [38-40]. Note that the stabilizing term introduces an extra error of order $O(\delta t)$ in a small region near the interface, but this error is of the same order as the splitting error introduced by treating $f(\phi), g_{1}(\boldsymbol{d})$ explicitly. So, the overall truncation error is essentially of the same order with or without the stabilizing term. An alternative way to discretize these two nonlinear functions is the so-called convex splitting approach $[16,37,51]$, but that involves the nonlinear scheme which needs efficient iterative methods to solve with the same truncated errors as the stabilized approach adopted in this paper.

- Inspired by the works in $[3,33]$, which deal with phase-field models of three-phase viscous fluids, we introduce new, explicit, convective velocities $\boldsymbol{u}_{\star}^{n}$ and $\boldsymbol{u}_{\star \star}^{n}$ in the phase equation and director field equation, respectively. $\boldsymbol{u}_{\star}^{n}$ and $\boldsymbol{u}_{\star \star}^{n}$ can be computed directly 
from (3.17) and (3.21) as follows:

$$
\begin{aligned}
& \boldsymbol{u}_{\star}^{n}=\left(I+\frac{\delta t}{M_{1}} \nabla \boldsymbol{d}^{n} \nabla \boldsymbol{d}^{n}\right)^{-1}\left(\boldsymbol{u}^{n}-\frac{\boldsymbol{d}^{n+1}-\boldsymbol{d}^{n}}{M_{1}} \nabla \boldsymbol{d}^{n}\right), \\
& \boldsymbol{u}_{\star \star}^{n}=\left(1+\frac{\delta t}{M_{2}} \nabla \phi^{n} \nabla \phi^{n}\right)^{-1}\left(\boldsymbol{u}_{\star}^{n}-\frac{\phi^{n+1}-\phi^{n}}{M_{2}} \nabla \phi^{n}\right) .
\end{aligned}
$$

It is easy to show $\operatorname{det}(I+c \nabla \boldsymbol{d} \nabla \boldsymbol{d})=1+c|\nabla \boldsymbol{d}|^{2}$ and $\operatorname{det}(I+c \nabla \phi \nabla \phi)=1+c|\nabla \phi|^{2}$, thus the above two matrices are indeed invertible. Notice that we substitute (3.26) into (3.14) and (3.27) into (3.18), respectively. After $\boldsymbol{d}^{n+1}, \phi^{n+1}$ are obtained, we calculate $\boldsymbol{u}_{\star}^{n}, \boldsymbol{u}_{\star \star}^{n}$ respectively.

- The scheme given in (3.14)-(3.25) is a totally decoupled, linear scheme. Indeed, (3.14), (3.18) and (3.22) are respectively (decoupled) linear elliptic equations for $\boldsymbol{d}^{n+1}, \phi^{n+1}$ and $\tilde{\boldsymbol{u}}^{n+1}$, and (3.24) can be reformulated as a Poisson equation for $p^{n+1}-p^{n}$. Therefore, at each time step, one only needs to solve a sequence of decoupled elliptic equations, which can be solved very efficiently using fast solvers.

We summarize the energy stability result in the following theorem.

Theorem 3.1. The scheme given in (3.14)-(3.25) admits a unique solution. Furthermore, If the following conditions hold,

$$
C^{n} \geq \frac{K L_{G}}{2}+\frac{K}{\eta^{2}}\left\|\frac{1-\phi^{n}}{2}\right\|_{\infty}, \quad S^{n} \geq \frac{\lambda L_{F}}{2},
$$

there exists the following semi-discrete energy dissipation law

$$
E^{n+1}+\frac{\delta t^{2}}{2}\left\|\nabla p^{n+1}\right\|^{2} \leq E^{n}+\frac{\delta t^{2}}{2}\left\|\nabla p^{n}\right\|^{2}-\delta t\left(\nu\left\|\nabla \tilde{\boldsymbol{u}}^{n+1}\right\|^{2}+M_{2}\left\|\nabla \mu^{n+1}\right\|^{2}+\frac{1}{M_{1}}\left\|\dot{\boldsymbol{d}}^{n+1}\right\|^{2}\right),
$$

where

$$
\begin{aligned}
E^{n}=\frac{1}{2}\left\|\boldsymbol{u}^{n}\right\|^{2}+ & K\left(\frac{1}{2}\left\|\nabla \boldsymbol{d}^{n}\right\|^{2}+\left(G_{1}\left(\boldsymbol{d}^{n}\right), 1\right)+\frac{1}{\eta^{2}}\left(\frac{1}{2}\left|\boldsymbol{d}^{n}\right|^{2}, 1-\phi^{n}\right)\right) \\
& +\lambda\left(\frac{\varepsilon}{2}\left\|\nabla \phi^{n}\right\|^{2}+\left(F\left(\phi^{n}\right), 1\right)\right) .
\end{aligned}
$$

Proof. From the definition of $\boldsymbol{u}_{\star}^{n}$ in (3.17) and $\boldsymbol{u}_{\star \star}^{n}$ in (3.21), we derive (3.22) as follows

$$
\frac{\tilde{\boldsymbol{u}}^{n+1}-\boldsymbol{u}_{\star \star}^{n}}{\delta t}+\left(\boldsymbol{u}^{n} \cdot \nabla\right) \tilde{\boldsymbol{u}}^{n+1}-\nu \Delta \tilde{\boldsymbol{u}}^{n+1}+\nabla p^{n}=0 .
$$

Taking the inner-product of (3.30) with $2 \delta t \tilde{\boldsymbol{u}}^{n+1}$, we obtain,

$$
\left\|\tilde{\boldsymbol{u}}^{n+1}\right\|^{2}-\left\|\boldsymbol{u}_{\star \star}^{n}\right\|^{2}+\left\|\tilde{\boldsymbol{u}}^{n+1}-\boldsymbol{u}_{\star \star}^{n}\right\|^{2}+2 \nu \delta t\left\|\nabla \tilde{\boldsymbol{u}}^{n+1}\right\|^{2}+2 \delta t\left(\nabla p^{n}, \tilde{\boldsymbol{u}}^{n+1}\right)=0 .
$$

To deal with the pressure term, we take the inner product of (3.24) with $2 \delta t^{2} \nabla p^{n}$ to arrive at

$$
\delta t^{2}\left(\left\|\nabla p^{n+1}\right\|^{2}-\left\|\nabla p^{n}\right\|^{2}-\left\|\nabla p^{n+1}-\nabla p^{n}\right\|^{2}\right)=2 \delta t\left(\tilde{\boldsymbol{u}}^{n+1}, \nabla p^{n}\right) .
$$

Taking the inner product of (3.24) with $\boldsymbol{u}^{n+1}$, we obtain

$$
\left\|\boldsymbol{u}^{n+1}\right\|^{2}+\left\|\boldsymbol{u}^{n+1}-\tilde{\boldsymbol{u}}^{n+1}\right\|^{2}=\left\|\tilde{\boldsymbol{u}}^{n+1}\right\|^{2} .
$$

It follows from (3.24) directly that

$$
\delta t^{2}\left\|\nabla p^{n+1}-\nabla p^{n}\right\|^{2}=\left\|\tilde{\boldsymbol{u}}^{n+1}-\boldsymbol{u}^{n+1}\right\|^{2} .
$$


Combining (3.31)-(3.34), we obtain

$$
\begin{aligned}
\frac{1}{2}\left\|\boldsymbol{u}^{n+1}\right\|^{2}-\frac{1}{2}\left\|\boldsymbol{u}_{\star \star}^{n}\right\|^{2} & +\frac{1}{2}\left\|\tilde{\boldsymbol{u}}^{n+1}-\boldsymbol{u}_{\star \star}^{n}\right\|^{2} \\
& +\frac{1}{2} \delta t^{2}\left(\left\|\nabla p^{n+1}\right\|^{2}-\left\|\nabla p^{n}\right\|^{2}\right)+\nu \delta t\left\|\nabla \tilde{\boldsymbol{u}}^{n+1}\right\|^{2}=0 .
\end{aligned}
$$

Taking the inner product of (3.14) with $\frac{1}{\delta t}\left(\boldsymbol{d}^{n+1}-\boldsymbol{d}^{n}\right)$, we get

$$
\begin{aligned}
& \frac{1}{\delta t} C^{n}\left\|\boldsymbol{d}^{n+1}-\boldsymbol{d}^{n}\right\|^{2}+\frac{1}{M_{1}}\left\|\dot{\boldsymbol{d}}^{n+1}\right\|^{2}-\frac{1}{M_{1}}\left(\dot{\boldsymbol{d}}^{n+1}, \boldsymbol{u}_{\star}^{n} \cdot \nabla \boldsymbol{d}^{n}\right) \\
& \quad+\frac{K}{\delta t}\left(\frac{1}{2}\left\|\nabla \boldsymbol{d}^{n+1}\right\|^{2}-\frac{1}{2}\left\|\nabla \boldsymbol{d}^{n}\right\|^{2}+\frac{1}{2}\left\|\nabla \boldsymbol{d}^{n+1}-\nabla \boldsymbol{d}^{n}\right\|^{2}\right)+\frac{K}{\delta t}\left(g_{1}\left(\boldsymbol{d}^{n}\right), \boldsymbol{d}^{n+1}-\boldsymbol{d}^{n}\right) \\
& =\frac{-K}{\delta t \eta^{2}}\left(\left(1-\phi^{n}\right) \boldsymbol{d}^{n+1}, \boldsymbol{d}^{n+1}-\boldsymbol{d}^{n}\right) .
\end{aligned}
$$

By taking the inner product of (3.18) with $\mu_{2}^{n+1}$, we obtain

$$
\frac{1}{\delta t}\left(\phi^{n+1}-\phi^{n}, \mu_{2}^{n+1}\right)-\left(\boldsymbol{u}_{\star \star}^{n} \phi^{n}, \nabla \mu_{2}^{n+1}\right)=-M_{2}\left\|\nabla \mu_{2}^{n+1}\right\|^{2},
$$

By taking the inner product of (3.19) with $\frac{1}{\delta t}\left(\phi^{n+1}-\phi^{n}\right)$, we get

$$
\begin{gathered}
\frac{S^{n}}{\delta t}\left\|\phi^{n+1}-\phi^{n}\right\|^{2}-\frac{1}{\delta t}\left(\phi^{n+1}-\phi^{n}, \mu_{2}^{n+1}\right) \\
(3.38)+\frac{\lambda \varepsilon}{\delta t}\left(\frac{1}{2}\left\|\nabla \phi^{n+1}\right\|^{2}-\frac{1}{2}\left\|\nabla \phi^{n}\right\|^{2}+\frac{1}{2}\left\|\nabla \phi^{n+1}-\nabla \phi^{n}\right\|^{2}\right)+\frac{\lambda}{\delta t}\left(f\left(\phi^{n}\right), \phi^{n+1}-\phi^{n}\right) \\
=\frac{K}{\delta t \eta^{2}}\left(\frac{1}{2}\left|\boldsymbol{d}^{n+1}\right|^{2}, \phi^{n+1}-\phi^{n}\right) .
\end{gathered}
$$

Combing (3.36)-(3.38) and multiplying the result by $\delta t$, we arrive

$$
\begin{aligned}
& C^{n}\left\|\boldsymbol{d}^{n+1}-\boldsymbol{d}^{n}\right\|^{2}+S^{n}\left\|\phi^{n+1}-\phi^{n}\right\|^{2}+K\left(\frac{1}{2}\left\|\nabla \boldsymbol{d}^{n+1}\right\|^{2}-\frac{1}{2}\left\|\nabla \boldsymbol{d}^{n}\right\|^{2}+\frac{1}{2}\left\|\nabla \boldsymbol{d}^{n+1}-\nabla \boldsymbol{d}^{n}\right\|^{2}\right) \\
& +\lambda \varepsilon\left(\frac{1}{2}\left\|\nabla \phi^{n+1}\right\|^{2}-\frac{1}{2}\left\|\nabla \phi^{n}\right\|^{2}+\frac{1}{2}\left\|\nabla \phi^{n+1}-\nabla \phi^{n}\right\|^{2}\right) \\
& -\frac{\delta t}{M_{1}}\left(\dot{\boldsymbol{d}}^{n+1}, \boldsymbol{u}_{\star} \cdot \nabla \boldsymbol{d}^{n}\right)-\delta t\left(\boldsymbol{u}_{\star \star}^{n} \phi^{n}, \nabla \mu_{2}^{n+1}\right) \\
& =-M_{2} \delta t\left\|\nabla \mu_{2}^{n+1}\right\|^{2}-\delta t \frac{1}{M_{1}}\left\|\dot{\boldsymbol{d}}^{n+1}\right\|^{2}-K\left(g_{1}\left(\boldsymbol{d}^{n}\right), \boldsymbol{d}^{n+1}-\boldsymbol{d}^{n}\right)-\lambda\left(f\left(\phi^{n}\right), \phi^{n+1}-\phi^{n}\right) \\
& -\frac{K}{\eta^{2}}\left(\left(1-\phi^{n}\right) \boldsymbol{d}^{n+1}, \boldsymbol{d}^{n+1}-\boldsymbol{d}^{n}\right)+\frac{K}{\eta^{2}}\left(\frac{1}{2}\left|\boldsymbol{d}^{n+1}\right|^{2}, \phi^{n+1}-\phi^{n}\right) .
\end{aligned}
$$

Taking the inner product of (3.17) with $\boldsymbol{u}_{\star}^{n}$, we obtain

$$
\frac{1}{2}\left\|\boldsymbol{u}_{\star}^{n}\right\|^{2}-\frac{1}{2}\left\|\boldsymbol{u}^{n}\right\|^{2}+\frac{1}{2}\left\|\boldsymbol{u}_{\star}^{n}-\boldsymbol{u}^{n}\right\|^{2}+\delta t \frac{1}{M_{1}}\left(\dot{\boldsymbol{d}}^{n+1} \nabla \boldsymbol{d}^{n}, \boldsymbol{u}_{\star}^{n}\right)=0 .
$$

Taking the inner product of (3.21) with $\boldsymbol{u}_{\star \star}^{n}$, we obtain

$$
\frac{1}{2}\left\|\boldsymbol{u}_{\star \star}^{n}\right\|^{2}-\frac{1}{2}\left\|\boldsymbol{u}_{\star}^{n}\right\|^{2}+\frac{1}{2}\left\|\boldsymbol{u}_{\star \star}^{n}-\boldsymbol{u}_{\star}^{n}\right\|^{2}+\delta t\left(\phi^{n} \nabla \mu_{2}^{n+1}, \boldsymbol{u}_{\star \star}^{n}\right)=0 .
$$


By combining (3.35), (3.39), (3.40), and (3.41), we obtain

$$
\begin{aligned}
\frac{1}{2}\left\|\boldsymbol{u}^{n+1}\right\|^{2}- & \frac{1}{2}\left\|\boldsymbol{u}^{n}\right\|^{2}+\frac{1}{2}\left\|\tilde{\boldsymbol{u}}^{n+1}-\boldsymbol{u}_{\star \star}^{n}\right\|^{2}+\frac{1}{2}\left\|\boldsymbol{u}_{\star \star}^{n}-\boldsymbol{u}_{\star}^{n}\right\|^{2}+\frac{1}{2}\left\|\boldsymbol{u}_{\star}^{n}-\boldsymbol{u}^{n}\right\|^{2} \\
& +\frac{1}{2} \delta t^{2}\left(\left\|\nabla p^{n+1}\right\|^{2}-\left\|\nabla p^{n}\right\|^{2}\right)+\nu \delta t\left\|\nabla \tilde{\boldsymbol{u}}^{n+1}\right\|^{2} \\
& +K\left(\frac{1}{2}\left\|\nabla \boldsymbol{d}^{n+1}\right\|^{2}-\frac{1}{2}\left\|\nabla \boldsymbol{d}^{n}\right\|^{2}+\frac{1}{2}\left\|\nabla \boldsymbol{d}^{n+1}-\nabla \boldsymbol{d}^{n}\right\|^{2}\right) \\
& +\lambda\left(\frac{\varepsilon}{2}\left\|\nabla \phi^{n+1}\right\|^{2}-\frac{\varepsilon}{2}\left\|\nabla \phi^{n}\right\|^{2}+\frac{\varepsilon}{2}\left\|\nabla \phi^{n+1}-\nabla \phi^{n}\right\|^{2}\right) \\
& +C^{n}\left\|\boldsymbol{d}^{n+1}-\boldsymbol{d}^{n}\right\|^{2}+S^{n}\left\|\phi^{n+1}-\phi^{n}\right\|^{2} \\
=-M_{2} \delta t \| & \mu_{2}^{n+1}\left\|^{2}-\delta t \frac{1}{M_{1}}\right\| \dot{\boldsymbol{d}}^{n+1} \|^{2} \\
& -K\left(g_{1}\left(\boldsymbol{d}^{n}\right), \boldsymbol{d}^{n+1}-\boldsymbol{d}^{n}\right)-\lambda\left(f\left(\phi^{n}\right), \phi^{n+1}-\phi^{n}\right) \\
& -\frac{K}{\eta^{2}}\left(\left(\left(1-\phi^{n}\right) \boldsymbol{d}^{n+1}, \boldsymbol{d}^{n+1}-\boldsymbol{d}^{n}\right)-\left(\frac{1}{2}\left|\boldsymbol{d}^{n+1}\right|^{2}, \phi^{n+1}-\phi^{n}\right)\right) .
\end{aligned}
$$

For the first two terms on the right hand side in (3.42), from a Taylor expansion, we have

$$
\begin{aligned}
-K\left(g_{1}\left(\boldsymbol{d}^{n}\right), \boldsymbol{d}^{n+1}-\boldsymbol{d}^{n}\right)= & -K\left(G_{1}\left(\boldsymbol{d}^{n+1}\right)-G_{1}\left(\boldsymbol{d}^{n}\right), 1\right) \\
& -K\left(\frac{1}{2} H_{G_{1}}(\boldsymbol{\theta})\left(\boldsymbol{d}^{n+1}-\boldsymbol{d}^{n}\right), \boldsymbol{d}^{n+1}-\boldsymbol{d}^{n}\right),
\end{aligned}
$$

and

$$
(3.44)-\lambda\left(f\left(\phi^{n}\right), \phi^{n+1}-\phi^{n}\right)=-\lambda\left(F\left(\phi^{n+1}\right)-F\left(\phi^{n}\right), 1\right)-\lambda\left(\frac{1}{2} f^{\prime}(\boldsymbol{\vartheta}),\left(\phi^{n+1}-\phi^{n}\right)^{2}\right) .
$$

The last two terms on the right hand side of (3.42) can be handled as follows.

$$
\begin{aligned}
& \left(\left(1-\phi^{n}\right) \boldsymbol{d}^{n+1}, \boldsymbol{d}^{n+1}-\boldsymbol{d}^{n}\right)-\left(\frac{1}{2}\left|\boldsymbol{d}^{n+1}\right|^{2}, \phi^{n+1}-\phi^{n}\right) \\
& (3.45)\left(\left(1-\phi^{n}\right), \frac{1}{2}\left|\boldsymbol{d}^{n+1}\right|^{2}-\frac{1}{2}\left|\boldsymbol{d}^{n}\right|^{2}+\frac{1}{2}\left|\boldsymbol{d}^{n+1}-\boldsymbol{d}^{n}\right|^{2}\right)+\left(\frac{1}{2}\left|\boldsymbol{d}^{n+1}\right|^{2},\left(1-\phi^{n+1}\right)-\left(1-\phi^{n}\right)\right) \\
& \quad=\left(\frac{1}{2}\left|\boldsymbol{d}^{n+1}\right|^{2},\left(1-\phi^{n+1}\right)\right)-\left(\frac{1}{2}\left|\boldsymbol{d}^{n}\right|^{2},\left(1-\phi^{n}\right)\right)+\left(\frac{1}{2}\left|\boldsymbol{d}^{n+1}-\boldsymbol{d}^{n}\right|^{2},\left(1-\phi^{n}\right)\right) .
\end{aligned}
$$

By combining (3.42), (3.43), (3.44) and (3.45), we obtain

$$
\begin{aligned}
E^{n+1}+ & \frac{1}{2} \delta t^{2}\left\|\nabla p^{n+1}\right\|^{2}+C^{n}\left\|\boldsymbol{d}^{n+1}-\boldsymbol{d}^{n}\right\|^{2}+S^{n}\left\|\phi^{n+1}-\phi^{n}\right\|^{2} \\
\leq & E^{n}-M_{2} \delta t\left\|\nabla \mu_{2}^{n+1}\right\|^{2}-\delta t \frac{1}{M_{1}}\left\|\dot{\boldsymbol{d}}^{n+1}\right\|^{2}-\frac{K}{\eta^{2}}\left(\frac{1}{2}\left|\boldsymbol{d}^{n+1}-\boldsymbol{d}^{n}\right|^{2},\left(1-\phi^{n}\right)\right) \\
& -K\left(\frac{1}{2} H_{G_{1}}(\boldsymbol{\theta})\left(\boldsymbol{d}^{n+1}-\boldsymbol{d}^{n}\right), \boldsymbol{d}^{n+1}-\boldsymbol{d}^{n}\right)-\lambda\left(\frac{1}{2} f^{\prime}(\boldsymbol{\vartheta}),\left(\phi^{n+1}-\phi^{n}\right)^{2}\right) .
\end{aligned}
$$


It is obvious that the last two terms are bounded as follows:

$$
\begin{aligned}
& -\frac{K}{\eta^{2}}\left(\frac{1}{2}\left|\boldsymbol{d}^{n+1}-\boldsymbol{d}^{n}\right|^{2},\left(1-\phi^{n}\right)\right) \leq \frac{K}{\eta^{2}}\left\|\frac{1-\phi^{n}}{2}\right\|_{\infty}\left\|\boldsymbol{d}^{n+1}-\boldsymbol{d}^{n}\right\|^{2}, \\
& -K\left(\frac{1}{2} H_{G_{1}}(\boldsymbol{\theta})\left(\boldsymbol{d}^{n+1}-\boldsymbol{d}^{n}\right), \boldsymbol{d}^{n+1}-\boldsymbol{d}^{n}\right) \leq \frac{K L_{G}}{2}\left\|\boldsymbol{d}^{n+1}-\boldsymbol{d}^{n}\right\|^{2}, \\
& -\lambda\left(\frac{1}{2} f^{\prime}(\boldsymbol{\vartheta}),\left(\phi^{n+1}-\phi^{n}\right)^{2}\right) \leq \frac{\lambda L_{F}}{2}\left\|\phi^{n+1}-\phi^{n}\right\|^{2} .
\end{aligned}
$$

Taking into account (3.28), we notice that these terms are absorbed into the stabilizing terms. Then, we obtain the desired result.

The uniqueness of the solution of the linear, discrete system follows from the energy decay law.

3.2. Nonlinear, Coupled, Semi-Discrete scheme for the full model. In this section, we present a nonlinear, coupled scheme to solve the full model (2.18)-(2.22) as follows.

Given the initial condition $\boldsymbol{d}^{0}, \boldsymbol{u}^{0}$ and $p^{0}=0$ and having computed $\left(\boldsymbol{d}^{n}, \boldsymbol{u}^{n}, p^{n}, \phi^{n}\right)$, we calculate $\left(\boldsymbol{d}^{n+1}, \tilde{\boldsymbol{u}}^{n+1}, \boldsymbol{u}^{n+1}, p^{n+1}, \phi^{n+1}\right)$ by the following two steps,

Step 1: Solve for $\left(\boldsymbol{d}^{n+1}, \phi^{n+1}, \tilde{\boldsymbol{u}}^{n+1}\right)$ :

$$
\begin{aligned}
& C^{n}\left(\boldsymbol{d}^{n+1}-\boldsymbol{d}^{n}\right)+\frac{1}{M_{1}} \dot{\boldsymbol{d}}^{n+1}=K\left(\Delta \boldsymbol{d}^{n+1}-g_{1}\left(\boldsymbol{d}^{n}\right)\right)-\frac{K}{\eta^{2}} \boldsymbol{d}^{n+1}\left(1-\phi^{n}\right) \\
& -\frac{A_{1}-A_{2}}{2}\left(\boldsymbol{d}^{n+1} \cdot \nabla \phi^{n+1}+\boldsymbol{d}^{n} \cdot \nabla \phi^{n}\right) \nabla \phi^{n+1}-A_{2}\left|\nabla \phi^{n}\right|^{2} \boldsymbol{d}^{n+1}, \\
& \frac{\phi^{n+1}-\phi^{n}}{\delta t}+\nabla \cdot\left(\tilde{\boldsymbol{u}}^{n+1} \phi^{n}\right)=M_{2} \Delta \mu_{2}^{n+1} \\
& S^{n}\left(\phi^{n+1}-\phi^{n}\right)+\mu_{2}^{n+1}=\lambda\left(-\varepsilon \Delta \phi^{n+1}+f\left(\phi^{n}\right)\right)-\frac{K}{2 \eta^{2}}\left|\boldsymbol{d}^{n+1}\right|^{2} \\
& +\frac{A_{2}-A_{1}}{2} \nabla \cdot\left(\left(\boldsymbol{d}^{n+1} \cdot \nabla \phi^{n+1}+\boldsymbol{d}^{n} \cdot \nabla \phi^{n}\right) \boldsymbol{d}^{n}\right)-A_{2} \nabla \cdot\left(\left|\boldsymbol{d}^{n+1}\right|^{2} \nabla \phi^{n+1}\right), \\
& \frac{\tilde{\boldsymbol{u}}^{n+1}-\boldsymbol{u}^{n}}{\delta t}+\left(\boldsymbol{u}^{n} \cdot \nabla\right) \tilde{\boldsymbol{u}}^{n+1}-\nu \Delta \tilde{\boldsymbol{u}}^{n+1}+\nabla p^{n}+\phi^{n} \nabla \mu_{2}^{n+1}+\frac{1}{M_{1}} \dot{\boldsymbol{d}}^{n+1} \nabla \boldsymbol{d}^{n} \\
& +\nabla \cdot\left(-\frac{a}{2}\left(\boldsymbol{d}^{n} \dot{\boldsymbol{d}}^{n+1}+\dot{\boldsymbol{d}}^{n+1} \boldsymbol{d}^{n}\right)+\frac{1}{2}\left(\boldsymbol{d}^{n} \dot{\boldsymbol{d}}^{n+1}-\dot{\boldsymbol{d}}^{n+1} \boldsymbol{d}^{n}\right)\right)=0,
\end{aligned}
$$

where

$$
\left\{\begin{array}{l}
\dot{\boldsymbol{d}}^{n+1}=\frac{\boldsymbol{d}^{n+1}-\boldsymbol{d}^{n}}{\delta t}+\tilde{\boldsymbol{u}}^{n+1} \cdot \nabla \boldsymbol{d}^{n}-\tilde{\boldsymbol{W}}^{n+1} \boldsymbol{d}^{n}-a \tilde{\boldsymbol{D}}^{n+1} \boldsymbol{d}^{n}, \\
\tilde{\boldsymbol{W}}^{n+1}=\frac{1}{2}\left(\nabla \tilde{\boldsymbol{u}}^{n+1}-\left(\nabla \tilde{\boldsymbol{u}}^{n+1}\right)^{T}\right), \quad \tilde{\boldsymbol{D}}^{n+1}=\frac{1}{2}\left(\nabla \tilde{\boldsymbol{u}}^{n+1}+\left(\nabla \tilde{\boldsymbol{u}}^{n+1}\right)^{T}\right),
\end{array}\right.
$$

and with boundary conditions

$$
\left.\tilde{\boldsymbol{u}}^{n+1}\right|_{\partial \Omega}=0,\left.\quad \frac{\partial \boldsymbol{d}^{n+1}}{\partial \boldsymbol{n}}\right|_{\partial \Omega}=0, \quad \nabla \phi^{n+1} \cdot \boldsymbol{n}=0, \quad \nabla \mu_{2}^{n+1} \cdot \boldsymbol{n}=0 .
$$

Step 2: Update $\boldsymbol{u}^{n+1}, p^{n+1}$ :

$$
\left\{\begin{array}{l}
\frac{1}{\delta t}\left(\boldsymbol{u}^{n+1}-\tilde{\boldsymbol{u}}^{n+1}\right)=-\nabla\left(p^{n+1}-p^{n}\right), \\
\nabla \cdot \boldsymbol{u}^{n+1}=0,\left.\quad \boldsymbol{u}^{n+1} \cdot \boldsymbol{n}\right|_{\partial \Omega}=0
\end{array}\right.
$$


Remark 3.2. The scheme given in (3.48)-(3.54) is nonlinear as we have terms like $\boldsymbol{d}^{n+1}$ coupled with $\phi^{n+1}$ in (3.48) and (3.50) due to anchoring effects, and $\left|\boldsymbol{d}^{n+1}\right|^{2}$ in (3.50). We note that it is a challenging issue to develop a linear decoupled (easy-to-implement) scheme to solve the full model (2.18)-(2.22) while maintaining the discrete energy dissipation laws, due to nonlinear anchoring energy and the coupling terms among $\boldsymbol{W}, \boldsymbol{D}$ and $\boldsymbol{d}$. The scheme (3.48)-(3.54) is the best one we can obtain at this point.

Nevertheless, we have a discrete energy dissipation law for this scheme as follows.

Theorem 3.2. Given (3.28), the scheme defined by (3.48)-(3.54) satisfies the following semidiscrete energy dissipation law:

$$
\begin{gathered}
E^{n+1}+\frac{\delta t^{2}}{2}\left\|\nabla p^{n+1}\right\|^{2}+\delta t\left(\nu\left\|\nabla \tilde{\boldsymbol{u}}^{n+1}\right\|^{2}+M_{2}\left\|\nabla \mu^{n+1}\right\|^{2}+\frac{1}{M_{1}}\left\|\dot{\boldsymbol{d}}^{n+1}\right\|^{2}\right) \\
\leq E^{n}+\frac{\delta t^{2}}{2}\left\|\nabla p^{n}\right\|^{2},
\end{gathered}
$$

where the semi-discrete energy $E^{n}$ is defined

$$
\begin{aligned}
E^{n}= & \frac{1}{2}\left\|\boldsymbol{u}^{n}\right\|^{2}+K\left(\frac{1}{2}\left\|\nabla \boldsymbol{d}^{n}\right\|^{2}+\left(G_{1}\left(\boldsymbol{d}^{n}\right), 1\right)+\frac{1}{\eta^{2}}\left(\frac{1}{2}\left|\boldsymbol{d}^{n}\right|^{2}, 1-\phi^{n}\right)\right) \\
& +\lambda\left(\frac{\varepsilon}{2}\left\|\nabla \phi^{n}\right\|^{2}+\left(F\left(\phi^{n}\right), 1\right)\right) \\
& +\left(\frac{A_{1}}{2}\left(\boldsymbol{d}^{n} \cdot \nabla \phi^{n}\right)^{2}+\frac{A_{2}}{2}\left(\left|\boldsymbol{d}^{n}\right|^{2}\left|\nabla \phi^{n}\right|^{2}-\left(\boldsymbol{d}^{n} \cdot \nabla \phi^{n}\right)^{2}\right), 1\right) .
\end{aligned}
$$

Proof. The proof is similar to that of Theorem 3.1, thus we only give a sketch of proof, highlighting the difference, mainly the terms induced from the frame-invariant derivative and anchoring energy and leaving the detailed proof to interested readers.

First of all, taking the inner-product of (3.51) with $\delta t \tilde{\boldsymbol{u}}^{n+1}$, we obtain,

$$
\begin{aligned}
\frac{1}{2}\left(\left\|\tilde{\boldsymbol{u}}^{n+1}\right\|^{2}-\right. & \left.\left\|\boldsymbol{u}^{n}\right\|^{2}+\left\|\tilde{\boldsymbol{u}}^{n+1}-\boldsymbol{u}^{n}\right\|^{2}\right)+\nu \delta t\left\|\nabla \tilde{\boldsymbol{u}}^{n+1}\right\|^{2}+\delta t\left(\nabla p^{n}, \tilde{\boldsymbol{u}}^{n+1}\right) \\
& +\delta t\left(\phi^{n} \nabla \mu_{2}^{n+1}, \tilde{\boldsymbol{u}}^{n+1}\right)+\frac{\delta t}{M_{1}}\left(\dot{\boldsymbol{d}}^{n+1} \nabla \boldsymbol{d}^{n}, \tilde{\boldsymbol{u}}^{n+1}\right)+ \\
& \left(-\frac{a}{2}\left(\boldsymbol{d}^{n} \dot{\boldsymbol{d}}^{n+1}+\dot{\boldsymbol{d}}^{n+1} \boldsymbol{d}^{n}\right)+\frac{1}{2}\left(\boldsymbol{d}^{n} \dot{\boldsymbol{d}}^{n+1}-\dot{\boldsymbol{d}}^{n+1} \boldsymbol{d}^{n}\right), \nabla \tilde{\boldsymbol{u}}^{n+1}\right)=0
\end{aligned}
$$

To deal with the pressure term, we take the inner product of (3.54) with $2 \delta t^{2} \nabla p^{n}$ to arrive at

$$
\delta t^{2}\left(\left\|\nabla p^{n+1}\right\|^{2}-\left\|\nabla p^{n}\right\|^{2}-\left\|\nabla p^{n+1}-\nabla p^{n}\right\|^{2}\right)=2 \delta t\left(\tilde{\boldsymbol{u}}^{n+1}, \nabla p^{n}\right) .
$$

Taking the inner product of (3.54) with $\boldsymbol{u}^{n+1}$, we obtain

$$
\left\|\boldsymbol{u}^{n+1}\right\|^{2}+\left\|\boldsymbol{u}^{n+1}-\tilde{\boldsymbol{u}}^{n+1}\right\|^{2}=\left\|\tilde{\boldsymbol{u}}^{n+1}\right\|^{2} .
$$

It follows from (3.54) that

$$
\delta t^{2}\left\|\nabla p^{n+1}-\nabla p^{n}\right\|^{2}=\left\|\tilde{\boldsymbol{u}}^{n+1}-\boldsymbol{u}^{n+1}\right\|^{2} .
$$


Combining (3.57)-(3.60), we obtain

$$
\begin{aligned}
\frac{1}{2}\left\|\boldsymbol{u}^{n+1}\right\|^{2}- & \frac{1}{2}\left\|\boldsymbol{u}^{n}\right\|^{2}+\frac{1}{2}\left\|\tilde{\boldsymbol{u}}^{n+1}-\boldsymbol{u}^{n}\right\|^{2}+\frac{1}{2} \delta t^{2}\left(\left\|\nabla p^{n+1}\right\|^{2}-\left\|\nabla p^{n}\right\|^{2}\right)+\nu \delta t\left\|\nabla \tilde{\boldsymbol{u}}^{n+1}\right\|^{2} \\
& +\delta t\left(\phi^{n} \nabla \mu_{2}^{n+1}, \tilde{\boldsymbol{u}}^{n+1}\right)+\frac{\delta t}{M_{1}}\left(\dot{\boldsymbol{d}}^{n+1} \nabla \boldsymbol{d}^{n}, \tilde{\boldsymbol{u}}^{n+1}\right) \\
& +\left(-\frac{a}{2}\left(\boldsymbol{d}^{n} \dot{\boldsymbol{d}}^{n+1}+\dot{\boldsymbol{d}}^{n+1} \boldsymbol{d}^{n}\right)+\frac{1}{2}\left(\boldsymbol{d}^{n} \dot{\boldsymbol{d}}^{n+1}-\dot{\boldsymbol{d}}^{n+1} \boldsymbol{d}^{n}\right), \nabla \tilde{\boldsymbol{u}}^{n+1}\right)=0 .
\end{aligned}
$$

Taking the inner product of (3.48) with $\left(\boldsymbol{d}^{n+1}-\boldsymbol{d}^{n}\right)$, we have

$$
\begin{gathered}
C^{n}\left\|\boldsymbol{d}^{n+1}-\boldsymbol{d}^{n}\right\|^{2}+\frac{\delta t}{M_{1}}\left\|\dot{\boldsymbol{d}}^{n+1}\right\|^{2}-\frac{\delta t}{M_{1}}\left(\dot{\boldsymbol{d}}^{n+1}, \tilde{\boldsymbol{u}}^{n+1} \cdot \nabla \boldsymbol{d}^{n}\right)+\delta t\left(\dot{\boldsymbol{d}}^{n+1}, \tilde{W}^{n+1} \boldsymbol{d}^{n}+a \tilde{\boldsymbol{D}}^{n+1} \boldsymbol{d}^{n}\right) \\
\begin{aligned}
(3.62) \quad & \frac{K}{2}\left(\left\|\nabla \boldsymbol{d}^{n+1}\right\|^{2}-\left\|\nabla \boldsymbol{d}^{n}\right\|^{2}+\left\|\nabla \boldsymbol{d}^{n+1}-\nabla \boldsymbol{d}^{n}\right\|^{2}\right)+K\left(g_{1}\left(\boldsymbol{d}^{n}\right), \boldsymbol{d}^{n+1}-\boldsymbol{d}^{n}\right) \\
+ & \frac{K}{\eta^{2}}\left(\left(1-\phi^{n}\right) \boldsymbol{d}^{n+1}, \boldsymbol{d}^{n+1}-\boldsymbol{d}^{n}\right)+\left(P 1, \boldsymbol{d}^{n+1}-\boldsymbol{d}^{n}\right)=0 .
\end{aligned}
\end{gathered}
$$

where

$$
P 1=\frac{A_{1}-A_{2}}{2}\left(\boldsymbol{d}^{n+1} \cdot \nabla \phi^{n+1}+\boldsymbol{d}^{n} \cdot \nabla \phi^{n}\right) \nabla \phi^{n+1}+A_{2}\left|\nabla \phi^{n}\right|^{2} \boldsymbol{d}^{n+1}
$$

Taking the inner product of (3.49) with $\delta t \mu_{2}^{n+1}$, we obtain

$$
\left(\phi^{n+1}-\phi^{n}, \mu_{2}^{n+1}\right)-\delta t\left(\tilde{\boldsymbol{u}}^{n+1} \phi^{n}, \nabla \mu_{2}^{n+1}\right)+\delta t M_{2}\left\|\nabla \mu_{2}^{n+1}\right\|^{2}=0,
$$

Taking the inner product of (3.50) with $\left(\phi^{n+1}-\phi^{n}\right)$, we get

$$
\begin{aligned}
& S^{n}\left\|\phi^{n+1}-\phi^{n}\right\|^{2}-\left(\phi^{n+1}-\phi^{n}, \mu_{2}^{n+1}\right) \\
& +\frac{\lambda \varepsilon}{2}\left(\left\|\nabla \phi^{n+1}\right\|^{2}-\left\|\nabla \phi^{n}\right\|^{2}+\left\|\nabla \phi^{n+1}-\nabla \phi^{n}\right\|^{2}\right)+\lambda\left(f\left(\phi^{n}\right), \phi^{n+1}-\phi^{n}\right) \\
& \quad-\frac{K}{\eta^{2}}\left(\frac{1}{2}\left|\boldsymbol{d}^{n+1}\right|^{2}, \phi^{n+1}-\phi^{n}\right)+\left(P 2, \phi^{n+1}-\phi^{n}\right)=0 .
\end{aligned}
$$

where

$$
P 2=-\frac{A_{2}-A_{1}}{2} \nabla \cdot\left(\left(\boldsymbol{d}^{n+1} \cdot \nabla \phi^{n+1}+\boldsymbol{d}^{n} \cdot \nabla \phi^{n}\right) \boldsymbol{d}^{n}\right)+A_{2} \nabla \cdot\left(\left|\boldsymbol{d}^{n+1}\right|^{2} \nabla \phi^{n+1}\right)
$$

It is readily verified that

$$
\left(P 1, \boldsymbol{d}^{n+1}-\boldsymbol{d}^{n}\right)+\left(P 2, \phi^{n+1}-\phi^{n}\right)=E_{a}^{n+1}-E_{a}^{n},
$$

where

$$
E_{a}^{n}=\left(\frac{A_{1}}{2}\left(\boldsymbol{d}^{n} \cdot \nabla \phi^{n}\right)^{2}+\frac{A_{2}}{2}\left(\left|\boldsymbol{d}^{n}\right|^{2}\left|\nabla \phi^{n}\right|^{2}-\left(\boldsymbol{d}^{n} \cdot \nabla \phi^{n}\right)^{2}\right), 1\right)
$$

and

$$
\begin{gathered}
\left(-\frac{a}{2}\left(\boldsymbol{d}^{n} \dot{\boldsymbol{d}}^{n+1}+\dot{\boldsymbol{d}}^{n+1} \boldsymbol{d}^{n}\right)+\frac{1}{2}\left(\boldsymbol{d}^{n} \dot{\boldsymbol{d}}^{n+1}-\dot{\boldsymbol{d}}^{n+1} \boldsymbol{d}^{n}\right), \nabla \tilde{\boldsymbol{u}}^{n+1}\right) \\
+\left(\dot{\boldsymbol{d}}^{n+1}, \tilde{W}^{n+1} \boldsymbol{d}^{n}+a \tilde{\boldsymbol{D}}^{n+1} \boldsymbol{d}^{n}\right)=0 .
\end{gathered}
$$

Notice that the equations $(3.61),(3.62),(3.64),(3.65)$ are similar to the ones in Theorem (3.1). Then, we end up with the same problem as in Theorem (3.1). 
Therefore, via (3.28), these terms are absorbed into the stabilizing terms; then, we obtain the desired result.

\section{Numerical Simulations}

In this section, we present some numerical examples to illustrate the usefulness of the proposed model (2.10) and the efficiency of the numerical scheme (3.48)-(3.54) in resolving interfacial dynamics in liquid crystal and viscous fluid mixtures. We also compute the simplified model (3.14)-(3.25), but the results show no essential differences with the full model for the model parameters used in this paper, where the flow field is relatively weak. We note a detailed parameter study is essential for investigating the physical properties of this LC model. However, in this paper, we focus on demonstrating the effectiveness of the new schemes and defer the detailed parameter study to the future.

4.1. Spatial discretization and GPU implementation. For the spatial operators in the scheme, we discretize them use second-order central finite difference methods over a uniform spatial grid, where the velocity fields are discretized at the center of the cell surface, and the pressure $p$, the phase variable and the director $(\phi$ and $\boldsymbol{d})$ are discretized at the cell center, shown in Figure 4.1. The boundary conditions are handled by the ghost cell method.

The fully discretized equations in the scheme are implemented on GPUs (graphic processing units) in 3 dimensional space for high-performance computing. To better utilize the performance of GPUs, we store all variables in the global memory and all parameters and mesh information (which do not change during the simulation) in the constant memory such that it significantly reduces the latency of data access.

One advantage of using GPUs is its virtual allocations of processors (we can claim as many thread labors as we desire even if it is beyond the existing number of multiprocessors on the physical device). Therefore, in our implementations, we allocate as many processors as the degrees of freedom allow.

4.2. Numerical examples. The $3 \mathrm{D}$ computational domain is set as follows: $\Omega=\left[0, L_{x}\right] \times$ $\left[0, L_{y}\right] \times\left[0, L_{z}\right]$, where $L_{x}, L_{y}, L_{z}$ are the domain lengths along the $x, y, z$ direction, respectively. We note that in the following numerical discussion, we use the scheme given by (3.48)-(3.51), as the full model is more relevant in term of predicting physical phenomena.

Example 1. Mesh refinement test. We first test the convergence rate of the new scheme in a $2 \mathrm{D}$ domain temporally. In order to eliminate the effect of spatial disturbances, we use $512 \times 512$ spatial grids. We modify the system of equations by adding forcing terms so that it admits the exact solution as follows:

$$
\left\{\begin{array}{l}
\phi(t, x, y)=\cos (t) \cos (\pi x) \cos (\pi y), \\
\boldsymbol{d}(t, x, y)=(\cos (2 \pi x) \sin (t), \sin (2 \pi x) \sin (t)), \\
\boldsymbol{u}(t, x, y)=\left(\pi \sin (2 \pi y) \sin ^{2}(\pi x) \sin (t),-\pi \sin (2 \pi x) \sin ^{2}(\pi y) \sin (t)\right), \\
p(t, x, y)=\cos (\pi x) \sin (\pi y) \sin (t) .
\end{array}\right.
$$

In Fig. 4.2, we show the $L^{1}, L^{2}$ and $L^{\infty}$ error at $t=1$ using the time step size $\delta t=2 \times 10^{-3}$, $10^{-3}, 5 \times 10^{-4}$, and $2.5 \times 10^{-4}$, for $\phi, \boldsymbol{d}$ and $\boldsymbol{u}$, respectively. The results show clearly that the convergence rate of the numerical scheme is at least first-order temporally.

Example 2. Director field orientation of a viscous drop in a nematic LC matrix. We simulate the steady state of a $2 \mathrm{D}$ viscous drop immersed in an ambient liquid crystal 


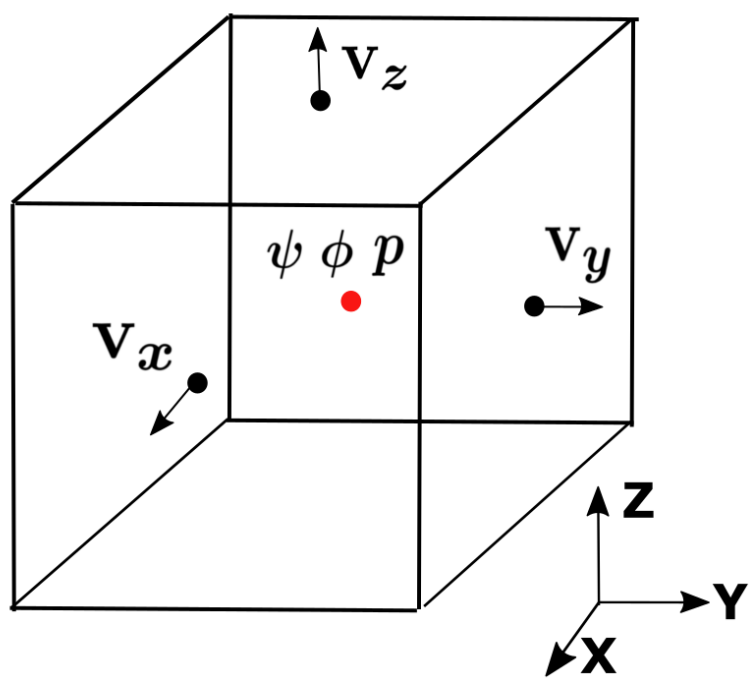

FigurE 4.1. Schematics for the discretization of the variables on the 3-D staggered grids.

matrix and plot the result in Figure 4.3, where $\phi=1$ (ambient fluid) indicates the nematic liquid crystal region, and $\phi=0$ (viscous drop) represents the viscous fluid. The domain size is $L_{x}=L_{y}=2$, the initial shape of the drop is a circular one with a uniform director field in the LC matrix,

$$
\begin{aligned}
& \phi(t=0)=0.5\left(1-\tanh \frac{\sqrt{(x-0.5)^{2}+(y-0.5)^{2}}-0.5}{0.01}\right) \\
& \boldsymbol{d}(t=0)=(0,1),
\end{aligned}
$$

We compare the drop shapes by varying the elastic constant $K$ with the same parallel anchoring constant $A_{1}=1, A_{2}=0$. Notice that the viscous drop forms a football shape eventually due to the larger distortional elastic effect of the ambient LC matrix. With a larger elastic parameter, the LC matrix has more elastic energy stored along the drop interface, which forces the drop interface to align along the director's direction. As the result, the drop deforms to the football shape in its final state. This simulation gives a qualitatively similar pattern reported in [62].

Example 3. Dynamical pinch-off of a falling liquid crystal drop. We simulate the dynamical process of a falling liquid crystal drop into an ambient viscous fluid matrix driven by gravity. The computational domain is chosen as $[0,0.25] \times[0,1] \times[0,0.25]$ in 3 -dimensional space. We use $128 \times 512 \times 128$ grid points in the simulation. The initial profile of the drop is defined via the phase variable $\phi, \boldsymbol{d}$ by

$$
\begin{aligned}
& \phi(t=0)=0.5\left(\tanh \frac{\sqrt{(x-1 / 8)^{2}+(y-1 / 2)^{2}+(z-1 / 8)^{2}}-0.1}{0.0025}+1\right), \\
& \boldsymbol{d}(t=0)=(0,1,0) .
\end{aligned}
$$

We consider the case where the density difference of the liquid crystal drop and ambient fluid is small so that we can use the Boussinesq approximation [29] in the momentum equation as 

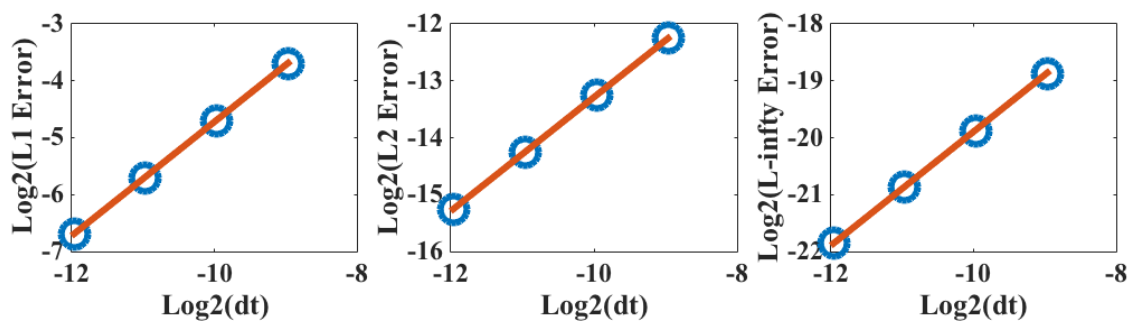

(A) The error plot for phase variable $\phi$.
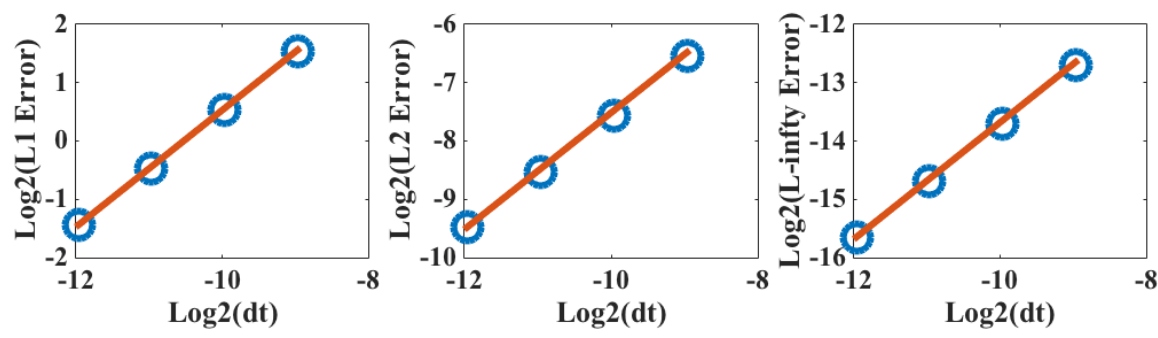

(в) The error plot for $d_{1}$.
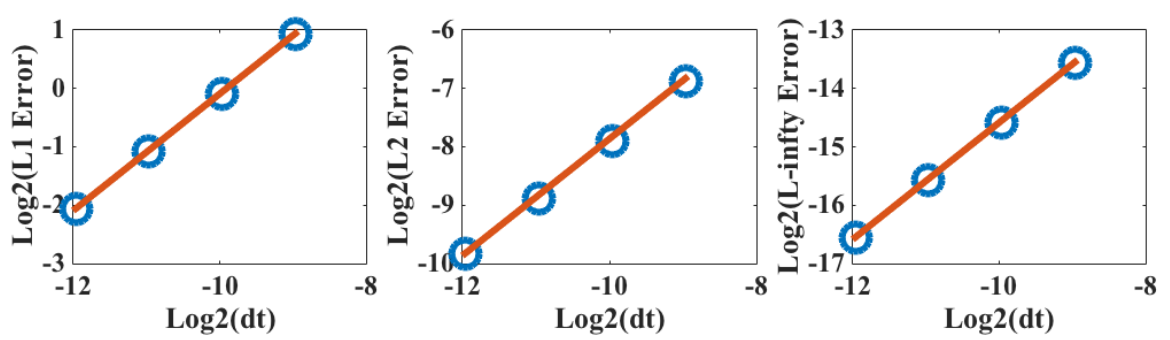

(C) The error plot for $d_{2}$.
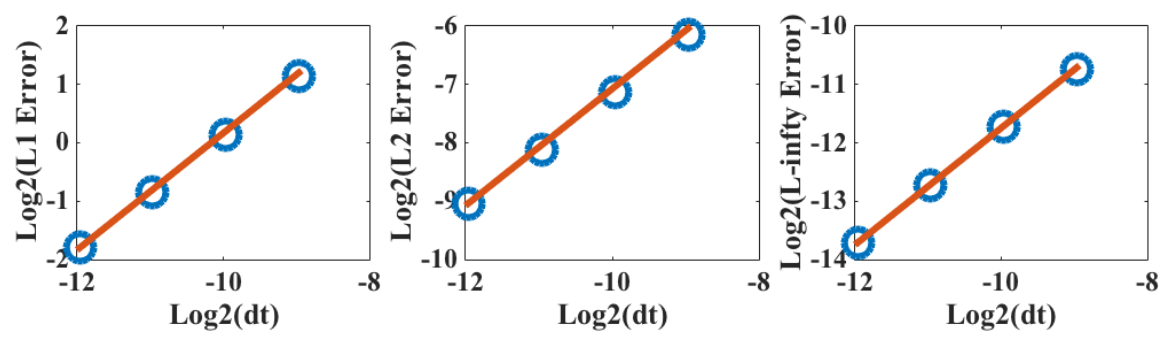

(D) The error plot for velocity component $u$.
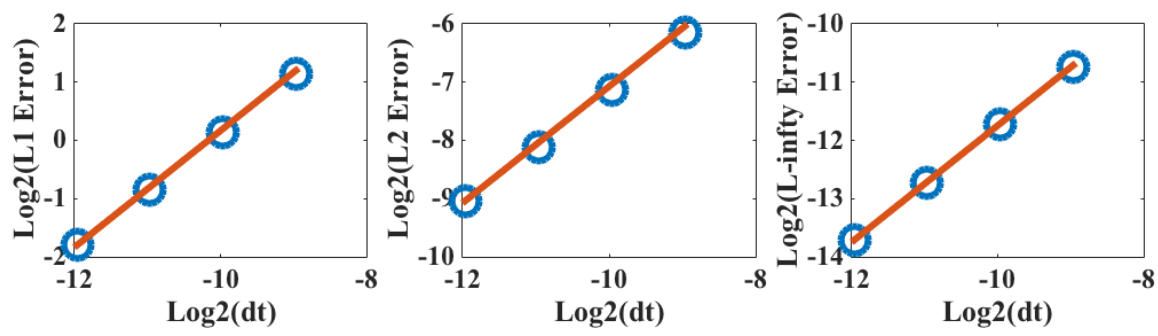

(E) The error plot for velocity component $v$.

FiguRE 4.2. Mesh refinement test for the convergence rate in time. The errors plotted are in the norm of $L_{1}, L_{2}, L_{\infty}$ for phase variable $\phi, \boldsymbol{d}=\left(d_{1}, d_{2}\right)$ and velocity field $\boldsymbol{u}=(u, v)$, respectively. The slopes of all error curves are close to 1 . 


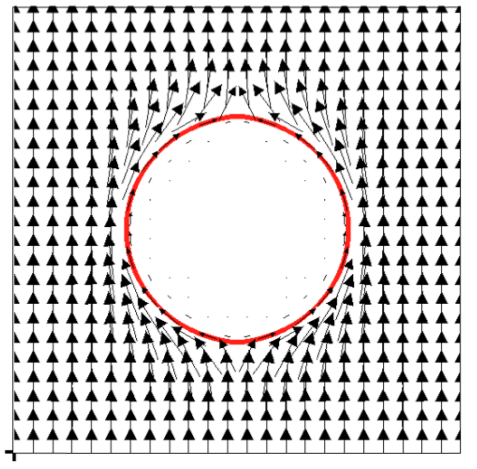

(A)

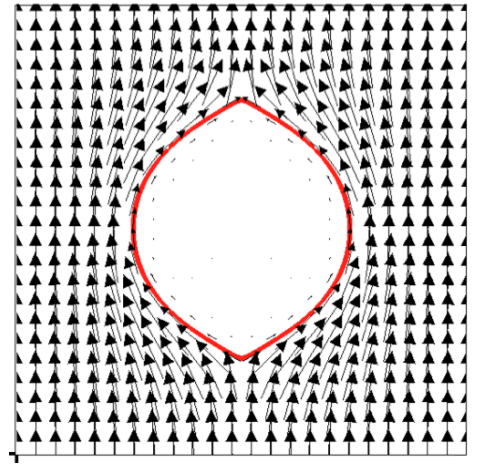

(B)

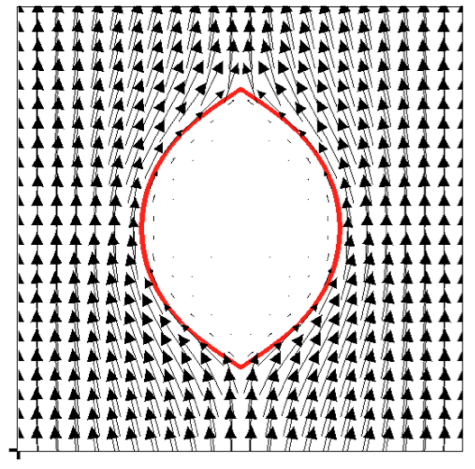

(C)

FIgURE 4.3. Steady states of a viscous drop immersed in a liquid crystal matrix at three selected values of the elastic constant $K$. (A) $K=10^{-3}$, (B) $K=10^{-2}$ and $(\mathrm{C}) K=10^{-1}$. The other parameter values are $\delta t=5 \times 10^{-4}, \lambda=0.1$, $\varepsilon=0.01, \eta=0.03, A_{1}=1, M_{1}=1$, and $M_{2}=10^{-6}$. The strong long-range elastic interaction characterized by a larger value of $K$ enhances the deformation of the viscous drop within the liquid crystal matrix.

follows:

$$
f=-\left(\left(\rho_{1}-\rho_{0}\right) \phi+\left(\rho_{2}-\rho_{0}\right)(1-\phi)\right) g,
$$

with $\rho_{1}=0.5, \rho_{2}=1.5, \rho_{0}=1$ and $g=500$. We set the anchoring constants $A_{1}=A_{2}=0$ for this simulation, i.e., there is no anchoring. Figure. 4.4 depicts dynamics of a falling LC drop. The elastic bulk energy leads to the formation of a persistent, long thread connecting the liquid crystal fluid at the faucet and the liquid crystal drop that forms at the leading edge of the falling drop. Instead of breaking up, the thin thread forms several smaller satellite drops as depicted in Figure. 4.4(G). Such simulations demonstrate the so called "bead-on-a-string" morphology associated with the falling liquid drop, which is qualitatively consistent with the experimental result in [48] and simulations in [41].

Example 4. Dynamics of a thinning liquid crystal filament due to capillary instability. We simulate the dynamical process of a thinning liquid crystal filament immersed in a viscous fluid matrix. The computational domain is chosen as $[0,0.25] \times[0,1] \times[0,0.25]$. We use $128 \times 512 \times 128$ grid points in the computation. The initial profile of the filament is defined via the phase variable $\phi, \boldsymbol{d}$ as

$$
\begin{aligned}
& \phi(t=0)=\frac{1}{2}\left(\tanh \left(\frac{R-r}{\varepsilon}\right)+1\right), \\
& \boldsymbol{d}(t=0)=(0,1,0),
\end{aligned}
$$

where

$$
\begin{aligned}
& r=\sqrt{(x-L x / 2)^{2}+(z-L z / 2)^{2}}, \\
& R=0.03+0.005 \sin (2(y-L y / 2)+0.5 \pi) .
\end{aligned}
$$

We set the anchoring condition at the interface to be parallel to the tangential direction along the axis of the liquid filament with $A_{1}=0.1$ and $A_{2}=0$. Figure 4.5 portraits the dynamical 


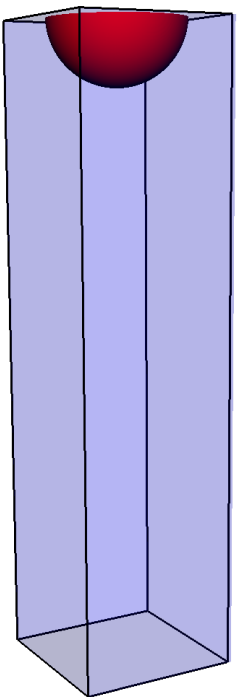

(A)

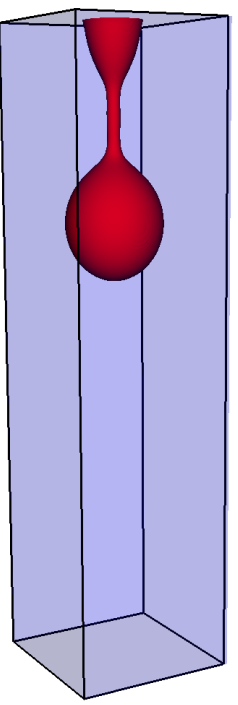

(E)

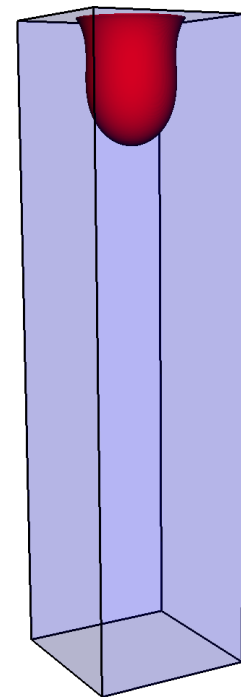

(B)

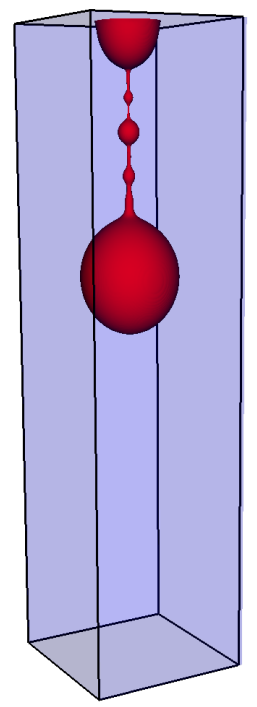

(F)

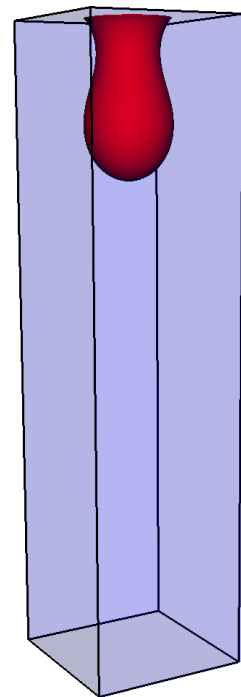

(C)

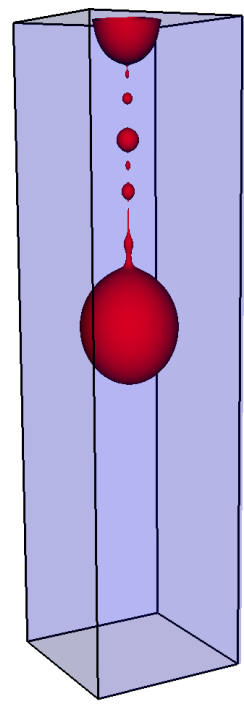

(G)

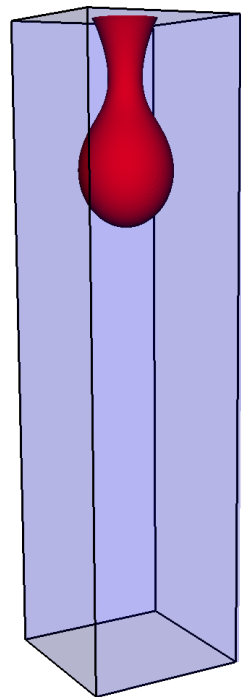

(D)

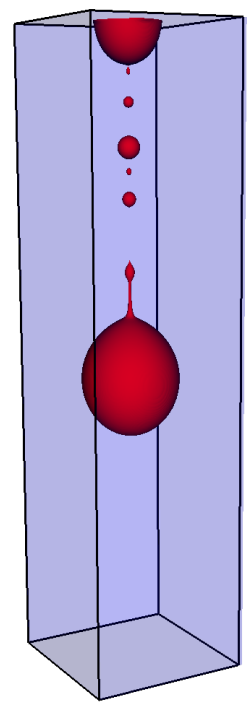

(H)

FiguRE 4.4. Snapshots of a dripping liquid crystal drop into a viscous fluid matrix at selected time slot $t=0,1,2,3,4,5,6,7,8$. The parameter values are $\delta t=5.0 \times 10^{-4}, \nu=1.0, M_{1}=10^{-10}, M_{2}=1.0, \gamma=9, \varepsilon=0.0025, K=0.01$, $\eta=0.01, \alpha_{2}=0.1$. 


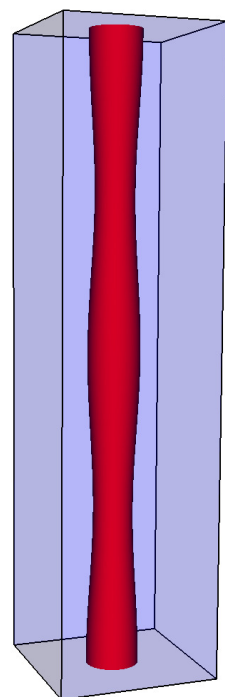

(A)

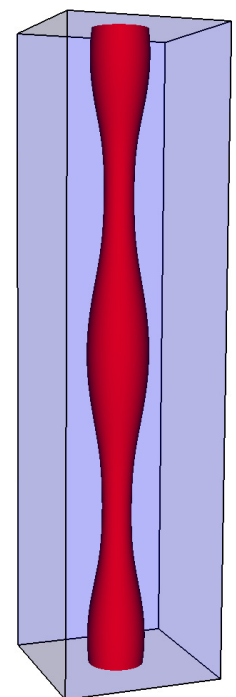

(B)

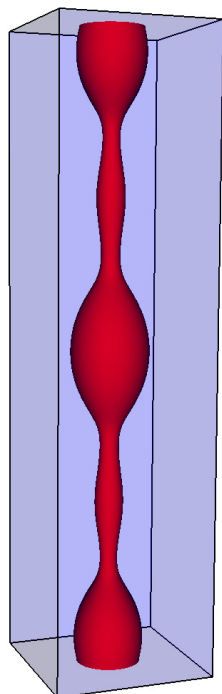

(c)

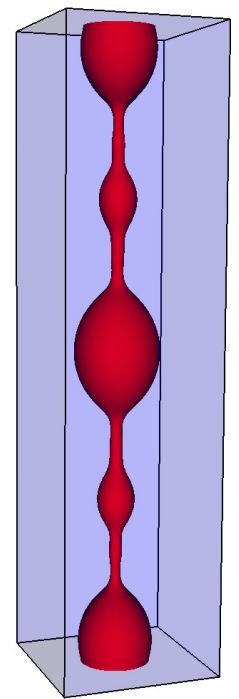

(D)

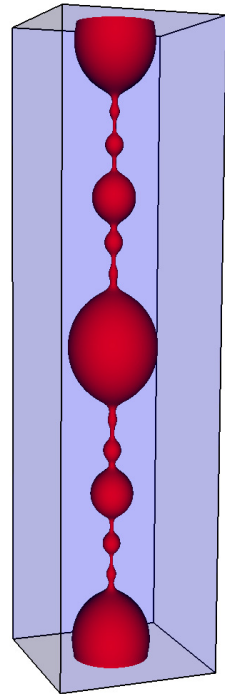

(E)

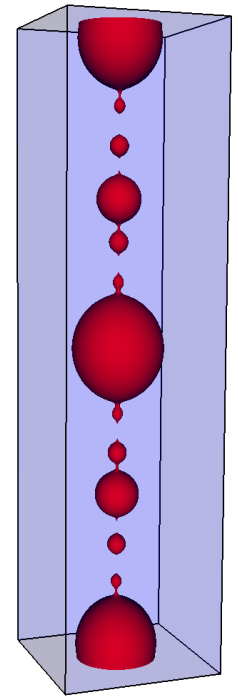

(F)

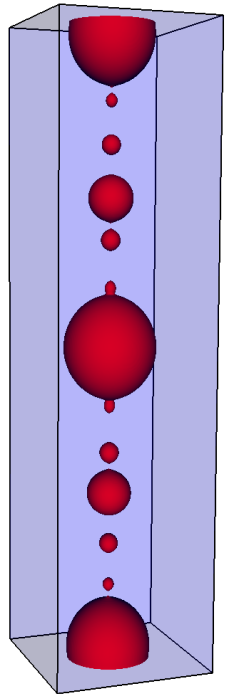

(G)

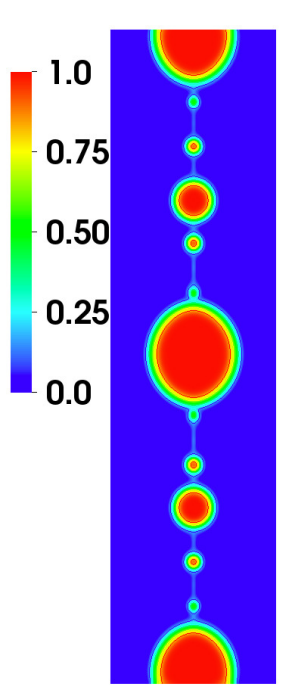

( $\mathrm{H})$

Figure 4.5. Snapshots of the dynamical process of a thinning liquid crystal filament . (A-F) liquid crystal filaments at timet $=0,2,4,5,6,7,8 ;(\mathrm{G}) 2 \mathrm{D}$ slice of liquid crystal filament at time $t=8$. The parameter values are $\delta t=5 \times 10^{-4}$, $\nu=1.0, M_{1}=10^{-10}, M_{2}=1, \lambda=9, \varepsilon=0.0025, K=0.01, \eta=0.01, A_{1}=0.1$. The filament eventually breaks into satellite drops. 
process of a thinning liquid crystal filament due to capillary instability. This figure shows the formation of beads induced by capillary instability. The initial filament is perturbed by a sinusoidal perturbation on the interface. The imposed perturbation grows, creating thin "necks" that continue to thin the filament (B-C). At $t=5((\mathrm{D})$ in the figure), three beads with different sizes are formed, which are connected by thin threads. The threads continue to thin and then many smaller beads appear on the filaments in (E). Eventually, the filament breaks up into satellite drops (F). This simulation yields qualitatively same patterns as reported in $[41,53]$.

\section{Concluding Remarks}

We have developed a novel phase field model for immiscible fluid mixtures of liquid crystals and viscous fluids, in which a simple yet efficient phase transition mechanism is employed to couple the liquid crystal phase with the viscous fluid phase. The model is derived using the variational principle coupled with the generalized Onsager principle so that it warrants energy dissipation. We then develop some energy stable schemes to solve the model. First, we devise a linear, decoupled scheme to solve a slightly simplified model which possesses the following properties: (i) it leads to completely decoupled elliptic equations to solve at each time step; (ii) it is unconditionally stable and obey a discrete energy law; and (iii) all elliptic equations are linear. Thus the scheme is very easy-to-implement. Then, we develop a fully couple energy stable scheme for the full model. We show that both schemes yield the same energy dissipation law at the discrete level consistent with the continuous one.

Although we consider only time discretized schemes in this study, the results can be carried over to any consistent finite-dimensional Galerkin approximations (finite elements or spectral) since the proofs are all based on a variational formulation with all test functions in the same space as the space of the trial functions. In this paper, we implement the numerical scheme for the full model using a finite difference method in space on GPUs for high performance computing. Three 2D and 3D numerical examples are given to illustrate the usefulness of the new model and the efficiency of the numerical schemes in resolving complex interfacial dynamics of the liquid crystal and viscous fluid mixture.

Acknowledgments. Q. Wang is partially supported by NSF grants DMS-1200487, DMS1517347, AFOSR FA9550-12-1-0178 and an SC EPSCOR GEAR award. X. Yang is partially supported by NSF grants DMS-1200487, NSF DMS-1418898, and AFOSR FA9550-12-1-0178.

J. Zhao and X. Yang thank the hospitality of Beijing Computational Science Research Center during their summer visits when this work was seeded.

\section{REFERENCES}

[1] Vera Joanne Alino, Jasmine Pang, and Kun-Lin Yang. Liquid crystal droplets as a hosting and sensing platform for developing immunoassays. Langmuir, 27:11784Ä̀̀-11789, 2011.

[2] A. N. Beris and B. Edwards. Thermodynamics of Flowing Systems. Ocford Science Publications, New York, 1994.

[3] Franck Boyer and Sebastian Minjeaud. Numerical schemes for a three component Cahn-Hilliard model. ESAIM. Mathematical Modelling and Numerical Analysis, 45(4):697-738, 2011.

[4] Luis A. Caffarelli and Nora E. Muler. An $L^{\infty}$ bound for solutions of the cahn-hilliard equation. Archive for Rational Mechanics and Analysis, 133(2):129-144, 1995. 
[5] L. Q. Chen and J. Shen. Applications of semi-implicit fourier-spectral method to phase field equations. Communications in Computational Physics, 108:147-158, 1998.

[6] R. Chen, G. Ji, X. Yang, and H. Zhang. Decoupled energy stable schemes for phase-field vesicle membrane model. Journal of Computational Physics, 302:509-523, 2015.

[7] N. Condette, C. Melcher, and E. Suli. Spectral approximation of pattern-forming nonlinear evolution equations with double-well potentials of quadratic growth. Mathematics of Computation, 80:205-223, 2011.

[8] J. W. Doane, A. Golemme, J. L. West, J. B. Whitehead Jr., and B.-G. Wu. Polymer dispersed liquid crystals for display application. Molecular Crystals and Liquid Crystals Incorporating Nonlinear Optics, 165:511-532, 1998.

[9] Q. Du, C. Liu, and X. Wang. A phase field approach in the numerical study of the elastic bending energy for vesicle membranes. Journal of Computational Physics, 198:450-468, 2004.

[10] Q. Du, C. Liu, and X. Wang. Simulating the deformation of vesicle membranes under elastic bending energy in three dimensions. J. Comput. Phys., 212:757-777, 2005.

[11] Qiang Du, Chun Liu, and Xiaoqiang Wang. A phase field approach in the numerical study of the elastic bending energy for vesicle membranes. Journal of Computational Physics, 198:450-468, 2004.

[12] K. R. Elder and Martin Grant. Modeling elastic and plastic deformations in nonequilibrium processing using phase field crystals. Phys. Rev. E., 70:051605, 2004.

[13] K. R. Elder, Mark Katakowski, Mikko Haataja, and Martin Grant. Modeling elasticity in crystal growth. Phys. Rev. Lett., 88(24):245701, 2002.

[14] J. L. Ericksen. Anisotropic fluids. Archive for Rational Mechanics and Analysis, 4:231-237, 1960.

[15] J. L. Ericksen. Liquid crystals with variable degree of orientation. IMA Preprint Series 559, 1989.

[16] David J. Eyre. Unconditionally gradient stable time marching the Cahn-Hilliard equation. In Computational and mathematical models of microstructural evolution (San Francisco, CA, 1998), volume 529 of Mater. Res. Soc. Sympos. Proc., pages 39-46. MRS, Warrendale, PA, 1998.

[17] J. J. Feng, C. Liu, J. Shen, and P. Yue. An energetic variational formulation with phase field methods for interfacial dynamics of complex fluids: advantages and challenges. IMA volumes in Mathematics and Applications, 140:1-26, 2005.

[18] J. L. Guermond, P. Minev, and J. Shen. An overview of projection methods for incompressible flows. Comput. Methods Appl. Mech. Engrg., 195:6011-6045, 2006.

[19] J. L. Guermond and J. Shen. On the error estimates of rotational pressure-correction projection methods. Mathematics of Computation, 73:1719-1737, 2004.

[20] M. E. Gurtin, D. Polignone, and J. Viñals. Two-phase binary fluids and immiscible fluids described by an order parameter. Math. Models Methods Appl. Sci., 6(6):815-831, 1996.

[21] J. Huang, F. Lin, and C. Wang. Regularity and existence of global solutions to the ericksen-leslie system in r2. Communications in Mathematical Physics, 331(2):805-850, 2014.

[22] D. Jacqmin. Diffuse interface model for incompressible two-phase flows with large density ratios. J. Comput. Phys., 155(1):96-127, 2007.

[23] D. Kessler, R. H. Nochetto, and A. Schmidt. A posteriori error control for the Allen-Cahn problem: circumventing Gronwall's inequality. Mathematical Modelling and Numerical Analysis, 38(1):129-142, 2004.

[24] M. Khan and S. Y. Park. Specific detection of avidin-biotin binding using liquid crystal droplets. Colloids Surf B Biointerfaces, 127:241-246, 2015.

[25] Junseok Kim. Phase-field models for multi-component fluid flows. Communication in Computational Physics, 12(3):613-661, 2012.

[26] F. M. Leslie. Some constitutive equations for anisotropic fluids. Q. Jl. Mech. Appl. Math., 19:357-370, 1966.

[27] F. Lin and C. Wang. Recent developments of analysis for hydrodynamic flow of nematic liquid crystals. Philosophical Transactions of the Royal Society of London Series A, 372:20130361, 2014.

[28] C. Liu and N. J. Walkington. Mixed methods for the approximation of liquid crystal flows. Mathematical Modeling and Numerical Analysis, 36(2):205-222, 2002.

[29] Chun Liu and Jie Shen. A phase field model for the mixture of two incompressible fluids and its approximation by a Fourier-spectral method. Physica D, 179(3-4):211-228, 2003.

[30] John S. Lowengrub, Andreas Ratz, and Axel Voigt. Phase field modeling of the dynamics of multicomponent vesicles spinodal decomposition coarsening budding and fission. Physical Review E, 79(3), 2009. 
[31] John S. Lowengrub and L. Truskinovsky. Quasi incompressible cahn-hilliard fluids and topological transitions. Proceedings of the Royal Society A, 454:2617-2654, 1998.

[32] C. Miehe, M. Hofacker, and F. Welschinger. A phase field model for rate-independent crack propagation: Robust algorithmic implementation based on operator splits. Computer Methods in Applied Mechanics and Engineering, 199:2765-2778, 2010.

[33] Sebastian Minjeaud. An unconditionally stable uncoupled scheme for a triphasic cahnilliard/navier stokes model. Numerical Methods for Partial Differential Equations, 29(2):584-618, 2013.

[34] Lars Onsager. Reciprocal relations in irreversible processes i. Physical Review, 37:405-426, 1931.

[35] Lars Onsager. Reciprocal relations in irreversible processes. ii. Physical Review, 38:2265-2279, 1931.

[36] L. Rayleigh. On the theory of surface forces-II. compressible fluids. Phil. Mag., 33, 1892.

[37] J. Shen, C. Wang, S. Wang, and X. Wang. Second-order convex splitting schemes for gradient flows with ehrlich-schwoebel type energy: application to thin film epitaxy. SIAM Journal of Numerical Analysis, 50(1):105-125, 2012.

[38] J. Shen and X. Yang. Energy stable schemes for cahn-hilliard phase-field model of two-phase incompressible flows. Chinese Ann. Math. series B, 31:743-758, 2010.

[39] J. Shen and X. Yang. Numerical approximation of allen-cahn and cahn-hilliard equations. Discrete and Continuous Dynamical Systems Series B, 28(4):1669-1691, 2010.

[40] J. Shen and X. Yang. A phase-field model and its numerical approximation for two-phase incompressible flows with different densities and viscositites. SIAM Journal of Scientific Computing, 32:1159-1179, 2010.

[41] J. Shen and X. Yang. Decoupled energy stable schems for phase field models of two phase complex fluids. SIAM Journal of Scientific Computing, 36(1):122-145, 2014.

[42] J. Shen and X. Yang. Decoupled energy stable schemes for phase field models of two phase incompressible flows. SIAM Journal of Numerical Analysis, 53(1):279-296, 2015.

[43] J. Shen, X. Yang, and H. Yu. Efficient energy stable numerical schemes for a phase field moving contact line model. Journal of Computational Physics, 284:617-630, 2015.

[44] Jie Shen. Modeling and numerical approximation of two-phase incompressible flows by a phase-field approach. Multiscale Modeling and Analysis for Materials Simulation, pages 147-196, 2011.

[45] Jie Shen, Xiaofeng Yang, and Qi Wang. Mass and volume conservation in phase field models for binary fluids. Communication in Computational Physics, 13(4):1045-1065, 2013.

[46] R. Spatschek, E. Brener, and A. Karma. A phase field model for rate-independent crack propagation: Robust algorithmic implementation based on operator splits. Philosophical Magazine, 91:75-95, 2010.

[47] Pengtao Sun, Chun Liu, and Jinchao Xu. Phase field model of thermo-induced marangoni effects in the mixtures and its numerical simulations with mixed finite element methods. Communications in Computational Physics, 6:1095-1117, 2009.

[48] V. Tirtaatmadja, G. H. McKinley, and J. J. Cooper-White. Drop formation and breakup of low viscosity elastic fluids: Effects of molecular weight and concentration. Phys. of Fluids., 18:043101, 2006.

[49] Elsen Tjhung, Davide Marenduzzo, and Michael E. Cates. Spontaneous symmetry breaking in active droplets provides a generic route to motility. PNAS, 109(31):12381-12386, 2012.

[50] J. van der Waals. The thermodynamic theory of capillarity under the hypothesis of a continuous density variation. J. Stat. Phys., 20:197-244, 1893.

[51] X. Xu, G. J. Van Zwieten, and K. G. van der Zee. Stabilized second-order convex splitting schemes for cahn-hilliard models with application to diffuse-interface tumor-growth models. International Journal for Numerical Methods in Biomedical Engineering, 30(180-203), 2014.

[52] X. Yang, J. J. Feng, C. Liu, and J. Shen. Numerical simulations of jet pinching-off and drop formation using an energetic variational phase-field method. Journal of Computational Physics, 218:417-428, 2006.

[53] X. Yang, M. G. Forest, H. Li, C. Liu, J. Shen, and Q. Wang. Modeling and simulations of drop pinch-off from liquid crystal filaments and the leaky liquid crystal faucet immsersed in viscous fluids. Journal of Computational Physics, 236:1-14, 2013.

[54] X. Yang, M. G. Forest, W. Mullins, and Q. Wang. 2-d lid-driven cavity flow of nematic polymers: an unsteady sea of defects. Soft Matter, 6:1138-1156, 2010.

[55] X. Yang, M. G. Forest, and Q. Wang. Near equilibrium dynamics and one-dimensional spatial-temporal structures of polar active liquid crystals. Chinese Physics B, 23(11), 2014. 
[56] X. Yang and Q. Wang. Capillary instability of axisymmetric active liquid crystal jets. Soft Matter, 10(35):6758-6776, 2014.

[57] Pengtao Yue, James J. Feng, Chun Liu, and Jie Shen. A diffuse interface method for simulating two phase flows of complex fluids. Journal of Fluid Mechanics, 515:293-317, 2004.

[58] Pengtao Yue, James J. Feng, Chun Liu, and Jie Shen. Diffuse interface simulations of drop coalescence and retraction in viscoelastic fluids. Journal of Non-Newtonian Fluid Mechanics, 129:163-176, 2005.

[59] Pengtao Yue, Chunfeng Zhou, James J. Feng, Carl F. Ollivier-Gooch, and Howard H. Hu. Phase field simulaitons of interfacial dynamics in viscoelastic fluids using finite elements with adaptive meshing. Journal of Computational Physics, 219:47-67, 2006.

[60] J. Zhao, X. Yang, J. Shen, and Q. Wang. A decoupled energy stable scheme for a hydrodynamic phase field model of mixtures of nematic liquid crystals and viscous fluids. Journal of Computational Physics, 305:539-556, 2016.

[61] Jia Zhao and Qi Wang. On energy stable schemes for a q tensor hydrodynamic model of liquid crystals. to be submitted, 2015.

[62] C. Zhou, P. Yue, J. Feng, C. Liu, and J. Shen. Heart-shaped bubbles rising in anisotropic liquids. Physics of Fluids, 19:041703, 2007.

[63] C. Zhou, P. Yue, and J. J. Feng. The rise of newtonian drops in a nematic liquid crystal. Journal of Fluid Mechanics, 593:385-404, 2007.

[64] C. Zhou, P. Yue, and J. J. Feng. Dynamic simulation of droplet interaction and self-assembly in a nematic liquid crystal. Langmuir, 24:3099-3110, 2008. 